\title{
DISCRETE AND CONFORMING SMOOTH DE RHAM COMPLEXES IN THREE DIMENSIONS
}

\author{
MICHAEL NEILAN
}

\begin{abstract}
Conforming discrete de Rham complexes consisting of finite element spaces with extra smoothness are constructed. In particular, we develop $H^{2}, \boldsymbol{H}^{1}$ (curl), $\boldsymbol{H}^{1}$ and $L^{2}$ conforming finite element spaces and show that an exactness property is satisfied. These results naturally lead to discretizations for Stokes and Brinkman type problems as well as conforming approximations to fourth order curl problems. In addition, we reduce the question of stability of the three-dimensional Scott-Vogelius finite element to a simply stated conjecture.
\end{abstract}

\section{INTRODUCTION}

The construction of finite element spaces that form a discrete de Rham complex is now a standard tool in the study of mixed finite element methods [1,2,11, 12, 21]. We recall that the de Rham complex in three dimensions with minimal smoothness measured in $L^{2}$ is given by

$$
\mathbb{R} \longrightarrow H^{1}(\Omega) \stackrel{\text { grad }}{\longrightarrow} \boldsymbol{H}(\mathbf{c u r l} ; \Omega) \stackrel{\text { curl }}{\longrightarrow} \boldsymbol{H}(\operatorname{div} ; \Omega) \stackrel{\text { div }}{\longrightarrow} L^{2}(\Omega) \longrightarrow 0,
$$

where $\boldsymbol{H}(\operatorname{curl} ; \Omega)(\operatorname{resp} ., \boldsymbol{H}(\operatorname{div} ; \Omega))$ is the space of square-integrable vector-valued functions whose curls (resp., divergence) are in $\boldsymbol{L}^{2}(\Omega)$ (resp., $L^{2}(\Omega)$ ). The precise definition of the notation used throughout the paper is given in the subsequent section. The statement that (1.1) is a complex simply means that the composition of two consecutive maps is zero. If the domain $\Omega$ is contractible and Lipschitz then the complex (1.1) is exact, that is, the range of each map is the kernel of the succeeding map. It is well known that many popular $H^{1}, \boldsymbol{H}(\mathbf{c u r l}), \boldsymbol{H}(\operatorname{div})$ and $L^{2}$ conforming finite element spaces form a discrete sub-complex of (1.1) $[1,2,21]$. Similar to the continuous setting, an exactness property of the sub-complex is satisfied.

In this paper, we develop and study discrete de Rham sub-complexes with additional smoothness. In particular, we derive conforming finite element spaces that mirror the complex 7,26

$$
\mathbb{R} \longrightarrow H^{2}(\Omega) \stackrel{\text { grad }}{\longrightarrow} \boldsymbol{H}^{1}(\operatorname{curl} ; \Omega) \stackrel{\text { curl }}{\longrightarrow} \boldsymbol{H}^{1}(\Omega) \stackrel{\text { div }}{\longrightarrow} L^{2}(\Omega) \longrightarrow 0 .
$$

This sequence is also exact (cf. Lemma 2.4 below). Our goal is to construct conforming finite element spaces $\Sigma_{h} \subset H^{2}(\Omega), \boldsymbol{\Upsilon}_{h} \subset \boldsymbol{H}^{1}(\operatorname{curl} ; \Omega), \boldsymbol{V}_{h} \subset \boldsymbol{H}^{1}(\Omega)$ and

Received by the editor May 30, 2013 and, in revised form, January 3, 2014.

2010 Mathematics Subject Classification. Primary 65N30, 65N12, 76M10.

Key words and phrases. Finite elements, de Rham complex, divergence free.

This work was supported in part by the National Science Foundation through grant number DMS-1115421. 
$Q_{h} \subset L^{2}(\Omega)$ with respect to a simplicial triangulation such that

$$
\mathbb{R} \stackrel{\subset}{\longrightarrow} \Sigma_{h} \stackrel{\text { grad }}{\longrightarrow} \Upsilon_{h} \stackrel{\text { curl }}{\longrightarrow} \boldsymbol{V}_{h} \stackrel{\text { div }}{\longrightarrow} Q_{h} \longrightarrow 0
$$

is an exact sub-complex of (1.2).

The construction of such finite element spaces naturally leads to discretizations for velocity-pressure formulations of Stokes and Brinkman-type problems [19,20, 28, where $\boldsymbol{V}_{h}$ and $Q_{h}$ are taken to be the finite element spaces for the velocity and pressure, respectively. The exactness property ensures that the divergence operator is surjective from $\boldsymbol{V}_{h}$ to $Q_{h}$. Along with a stability estimate, this result shows that the inf-sup/LBB condition is satisfied, and therefore the finite element pair $\boldsymbol{V}_{h} \times Q_{h}$ forms a stable pair [3,6]. In addition, the diagram (1.3) implies $\operatorname{div} \boldsymbol{V}_{h} \subseteq Q_{h}$ and therefore the resulting discretization produces exactly divergence-free velocity approximations. As far as we are aware, this is the first stable and conforming finite element pair consisting of polynomial basis functions that yield divergence-free velocities on general shape-regular triangulations in three dimensions (see $30,31,33$ for partial results on specific uniform triangulations, and the recent paper [17] where rational functions are used to obtain exactly divergence-free approximations). We remark that conforming finite spaces of the complex (1.1) with extra smoothness have been constructed in [26] leading to non-conforming approximations of the spaces appearing in (1.2). Their construction leads to non-conforming approximations for Stokes and Brinkman-type problems. Divergence-free velocity approximations have also been proposed by [9, 10] and [13 using discontinuous Galerkin and isogeometric methods, respectively.

The spaces $\boldsymbol{\Upsilon}_{h}$ can be utilized to compute conforming finite element approximations of magnetohydrodynamic equations with fourth-order terms [25, 34. Nonconforming finite element spaces of $\boldsymbol{H}^{1}(\mathbf{c u r l} ; \Omega)$ have been proposed in [26, 33], and a Ciarlet-type mixed finite element for fourth order curl problems was studied in [25]. Our construction appears to be the first appearance of $\boldsymbol{H}^{1}(\mathbf{c u r l} ; \Omega)$ conforming finite elements on tetrahedral meshes in the literature.

This work is motivated by the recent results in [15], where Falk and the author constructed analogous two-dimensional finite element spaces based on the complex

$$
\mathbb{R} \stackrel{\subset}{\longrightarrow} H^{2}(\Omega) \stackrel{\text { curl }}{\longrightarrow} \boldsymbol{H}^{1}(\Omega) \stackrel{\text { div }}{\longrightarrow} L^{2}(\Omega) \longrightarrow 0 .
$$

Here, the generalized Argyris space of degree $k+1(k \geq 4)$ is taken to be the $H^{2}(\Omega)$-conforming finite element space appearing in the sequence (1.4). This construction naturally leads to conforming and divergence-free Stokes elements with extra smoothness at vertices of the triangulation. In particular, the velocity and pressure elements constructed in [15] are $C^{1}$ and $C^{0}$, respectively, at vertices of the triangulation.

Similarly, we use the three-dimensional analogue of the Argyris element introduced by Zeníšek and Zhang [29,32] as our $H^{2}(\Omega)$-conforming element. This space consists of globally $C^{1}$ piecewise polynomials of degree $k+2$ (with $k \geq 7$ ) that are $C^{4}$ at vertices and $C^{2}$ at edges of the triangulation (cf. Section 3). The corresponding spaces $\boldsymbol{\Upsilon}_{h}, \boldsymbol{V}_{h}$ and $Q_{h}$ consist of piecewise polynomials of degree $k+1$, $k$ and $k-1(k \geq 6)$, respectively. In addition to the global regularity properties $\boldsymbol{\Upsilon}_{h} \subset \boldsymbol{H}^{1}(\operatorname{curl} ; \Omega), \boldsymbol{V}_{h} \subset \boldsymbol{H}^{1}(\Omega)$ and $Q_{h} \subset L^{2}(\Omega)$, the spaces also have enhanced regularity at sub-simplexes of the triangulation. For example, functions in $\boldsymbol{V}_{h}$ are $C^{2}$ at vertices and $C^{1}$ at edges of the triangulation (but only $C^{0}$ globally). Due 
to their relative high polynomial degree and complexity, the practical importance of these elements may be limited. Nonetheless, we believe that the framework and theory may shed new light into developing simpler methods (cf. Section 7).

The rest of the paper is organized as follows. In Section 2 we set the notation and state some preliminary results. In Section 3 we precisely state the definition of the finite element spaces. We then show that the discrete complex (1.3) is exact in Section 4 by a direct construction of Fortin operators. In Section 5 , we derive the approximation properties of the finite element spaces. In Section 6 we discuss the construction of the analogous spaces with homogeneous boundary conditions and its relation with the three-dimensional Scott-Vogelius element. Finally, we summarize our results and discuss possible extensions in Section 7.

\section{Preliminaries}

2.1. Notation. Throughout the paper we assume that the domain $\Omega$ is contractible with Lipschitz boundary $\partial \Omega$. For a set $D \subset \mathbb{R}^{d}$, we denote by $H^{m}(D)$ the Hilbert space consisting of square integrable functions whose distributional derivatives up to order $m$ are also square integrable. In the case $m=0$, we set $L^{2}(D)=H^{0}(D)$. The space $H_{0}^{m}(D)$ consists of all functions in $H^{m}(D)$ whose traces vanish up to order $m-1$, and the space of functions in $H^{m}(D)$ which have zero mean is denoted by $\stackrel{\circ}{H}^{m}(D)$. The corresponding vector-valued spaces are given by $\boldsymbol{H}^{m}(D), \boldsymbol{L}^{2}(D)$, and $\boldsymbol{H}_{0}^{m}(D)$. We also define (with $d=3$ )

$$
\begin{aligned}
\boldsymbol{H}(\operatorname{curl} ; D) & =\left\{\boldsymbol{v} \in \boldsymbol{L}^{2}(D): \operatorname{curl} \boldsymbol{v} \in \boldsymbol{L}^{2}(D)\right\}, \\
\boldsymbol{H}_{0}(\operatorname{curl} ; D) & =\left\{\boldsymbol{v} \in \boldsymbol{H}(\operatorname{curl} ; D): \boldsymbol{v} \times\left.\boldsymbol{n}_{D}\right|_{\partial D}=0\right\}, \\
\boldsymbol{H}^{1}(\operatorname{curl} ; D) & =\left\{\boldsymbol{v} \in \boldsymbol{H}^{1}(D): \operatorname{curl} \boldsymbol{v} \in \boldsymbol{H}^{1}(D)\right\}, \\
\boldsymbol{H}_{0}^{1}(\operatorname{curl} ; D) & =\left\{\boldsymbol{v} \in \boldsymbol{H}^{1}(\operatorname{curl} ; D) \cap \boldsymbol{H}_{0}^{1}(D): \boldsymbol{v} \times\left.\boldsymbol{n}_{D}\right|_{\partial D}=0\right\}, \\
\boldsymbol{H}(\operatorname{div} ; D) & =\left\{\boldsymbol{v} \in \boldsymbol{L}^{2}(D): \operatorname{div} \boldsymbol{v} \in L^{2}(D)\right\}, \\
\boldsymbol{H}_{0}(\operatorname{div} ; D) & =\left\{\boldsymbol{v} \in \boldsymbol{H}(\operatorname{div} ; D):\left.\boldsymbol{v} \cdot \boldsymbol{n}_{D}\right|_{\partial D}=0\right\}
\end{aligned}
$$

Here, $\boldsymbol{n}_{D}$ is the outward unit normal of the boundary $\partial D$. When the context is clear we shall simply write $\boldsymbol{n}$. The space of polynomials of degree less than or equal to $k$ on $D$ is given by $\mathcal{P}_{k}(D)$, and the analogous vector-valued space is $\mathcal{P}_{k}(D):=\left[\mathcal{P}_{k}(D)\right]^{d}$.

Let $\mathcal{T}_{h}$ be a shape-regular triangulation of the domain $\Omega$ [4,8, with $h_{T}=\operatorname{diam}(T)$ for all $T \in \mathcal{T}_{h}$ and $h=\max _{T \in \mathcal{T}_{h}} h_{T}$. We denote by $\mathcal{F}_{h}$ the set of faces in $\mathcal{T}_{h}$, and by $\mathcal{F}_{h}^{B}$ the set of boundary faces; that is, those faces satisfying $F \subset \partial \Omega$. The set of edges in the triangulation $\mathcal{T}_{h}$ is denoted by $\mathcal{E}_{h}$, and the set of vertices is denoted by $\mathcal{V}_{h}$. We define $h_{F}=\operatorname{diam}(F)$ and $h_{e}=\operatorname{diam}(e)$ for all $F \in \mathcal{F}_{h}$ and $e \in \mathcal{E}_{h}$. For a given tetrahedron $T \in \mathcal{T}_{h}$, let $\mathcal{F}_{h}(T), \mathcal{E}_{h}(T)$ and $\mathcal{V}_{h}(T)$ be, respectively, the set of four faces, six edges, and four vertices of $T$. We also set $\omega(T)=\sum_{T^{\prime} \in \mathcal{T}_{h}} \overline{\partial T \cap \overline{\partial T^{\prime}} \neq \emptyset} T^{\prime}$ to denote the "patch" of the element $T$.

Given $T \in \mathcal{T}_{h}$, we denote by $\left\{\lambda_{F}\right\}_{F \in \mathcal{F}_{h}(T)}$ the four barycentric coordinates of $T$ labeled such that $\left.\lambda_{F}\right|_{F}=0$ for all $F \in \mathcal{F}_{h}(T)$. The quartic volume bubble function on the simplex $T$ is defined as $b_{T}:=\prod_{F \in \mathcal{F}_{h}(T)} \lambda_{F}$, and the cubic face bubble function associated to the face $F$ is defined as $b_{F}:=\prod_{G \in \mathcal{F}_{h}(T) \backslash\{F\}} b_{G}$. It 
is then straight-forward to verify the identity

$$
\left.\operatorname{grad} b_{T}\right|_{F}=\left(\operatorname{grad} \lambda_{F}\right) b_{F}=-\left|\operatorname{grad} \lambda_{F}\right| \boldsymbol{n}_{F} b_{F} .
$$

Finally, the letter $C$ will denote a generic positive, $h$-independent constant that may take on different values at different occurrences in the paper.

2.2. Preliminary Results. With the notation set, we now state some preliminary results that will be used extensively in the analysis below. First, we state some standard trace inequalities and inverse estimates on a simplex.

Lemma 2.1. Let $T \in \mathcal{T}_{h}$ be a given simplex in the triangulation.

- For any $w \in H^{1}(T)$, there holds

$$
\sum_{F \in \mathcal{F}_{h}(T)}\|w\|_{L^{2}(F)}^{2} \leq C\left(h_{T}^{-1}\|w\|_{L^{2}(T)}^{2}+h_{T}\|w\|_{H^{1}(T)}^{2}\right) .
$$

- If $w \in H^{2}(T)$, there holds

$$
\sum_{e \in \mathcal{E}_{h}(T)}\|w\|_{L^{2}(e)}^{2} \leq C\left(h_{T}^{-2}\|w\|_{L^{2}(T)}^{2}+\|w\|_{H^{1}(T)}^{2}+h_{T}^{2}\|w\|_{H^{2}(T)}^{2}\right) .
$$

Proof. The first trace inequality (2.2) can be found in [4,8]. To obtain the second inequality, we observe that

$$
\begin{aligned}
\sum_{e \in \mathcal{E}_{h}(T)}\|v\|_{L^{2}(e)}^{2} & \leq C \sum_{e \in \mathcal{E}_{h}(T)} \sum_{\substack{F \in \mathcal{F}_{h}(T) \\
e \subset \partial F}}\left(h_{F}^{-1}\|v\|_{L^{2}(F)}^{2}+h_{F}\|v\|_{H^{1}(F)}^{2}\right) \\
& \leq C \sum_{F \in \mathcal{F}_{h}(T)}\left(h_{T}^{-1}\|v\|_{L^{2}(F)}^{2}+h_{T}\|v\|_{H^{1}(F)}^{2}\right) .
\end{aligned}
$$

Combining this last estimate with (2.2), we obtain (2.3).

Lemma $2.2([4,8])$. For any $w \in \mathcal{P}_{k}(T)$ and integers $m, n$ with $m \geq n$, there holds

$$
\|w\|_{H^{m}(T)} \leq C h_{T}^{n-m}\|w\|_{H^{n}(T)},
$$

where the constant $C>0$ depends on the shape regularity of $T, m, n$, and $k$, but is independent of $h_{T}$.

Lemma $2.3(24])$. For an integer $k$, let $\mathcal{P}_{k}$ denote the Lagrange finite element space consisting of globally continuous piecewise polynomials of degree $\leq k$. Then there exists an interpolation operator $I_{h}: H^{1}(\Omega) \rightarrow \mathcal{P}_{k}$ such that if $w \in H^{m}(\Omega)$ with $1 \leq m \leq k+1$,

$$
\left\|w-I_{h} w\right\|_{H^{s}(T)} \leq C h_{T}^{m-s}\|w\|_{H^{m}(\omega(T))} \quad 0 \leq s \leq m, \forall T \in \mathcal{T}_{h} .
$$

Lemma 2.4. The complex (1.2) is exact.

Proof. We first recall that (1.1) is an exact complex. Suppose $\boldsymbol{z} \in \boldsymbol{H}^{1}(\mathbf{c u r l} ; \Omega) \subset$ $\boldsymbol{H}(\operatorname{curl} ; \Omega)$ satisfies $\operatorname{curl} \boldsymbol{z}=0$. Then by (1.1) there exists $p \in H^{1}(\Omega)$ with $\boldsymbol{z}=$ $\operatorname{grad} p$. Since $\boldsymbol{z} \in \boldsymbol{H}^{1}(\Omega)$, we have $\operatorname{grad} p \in \boldsymbol{H}^{1}(\Omega)$ and therefore $p \in H^{2}(\Omega)$. Now if $\boldsymbol{v} \in \boldsymbol{H}^{1}(\Omega)$ satisfies $\operatorname{div} \boldsymbol{v}=0$, then by [16, Corollary 3.3] there exists $\boldsymbol{w} \in \boldsymbol{H}^{2}(\Omega) \subset \boldsymbol{H}^{1}(\operatorname{curl} ; \Omega)$ with $\boldsymbol{v}=\operatorname{curl} \boldsymbol{w}$. Finally, for any $q \in L^{2}(\Omega)$ there exists $\boldsymbol{r} \in \boldsymbol{H}^{1}(\Omega)$ such that $\operatorname{div} \boldsymbol{r}=q$ [16]. It then follows that (1.2) is exact. 
Next, we state an analogous result incorporating homogeneous boundary conditions. The proof can be found in [16, 26].

Lemma 2.5. If $D$ is convex, then the complex

$$
0 \longrightarrow H_{0}^{2}(D) \stackrel{\text { grad }}{\longrightarrow} \boldsymbol{H}_{0}^{1}(\operatorname{curl} ; D) \stackrel{\text { curl }}{\longrightarrow} \boldsymbol{H}_{0}^{1}(D) \stackrel{\text { div }}{\longrightarrow} \stackrel{\circ}{L^{2}}(D) \longrightarrow 0
$$

is exact.

\section{The Finite ELEMENT SPACES}

In this section, we define the finite element spaces $\Sigma_{h} \subset H^{2}(\Omega), \mathbf{\Upsilon}_{h} \subset \boldsymbol{H}^{1}(\mathbf{c u r l} ; \Omega)$, $\boldsymbol{V}_{h} \subset \boldsymbol{H}^{1}(\Omega)$ and $Q_{h} \subset L^{2}(\Omega)$ appearing in the sub-complex (1.3). Following Ciarlet's convention $[\underline{8}$, the definition of a finite space consists of three components: the triangulation $\mathcal{T}_{h}$, the local space of shape-functions, and the set of degrees of freedom (often abbreviated DOFs).

$H^{2}$-conforming finite element spaces. We take $\Sigma_{h}$ to be the $H^{2}$-conforming finite element space first proposed by A. Ženíšek 29] and later generalized by S. Zhang [32. This space consists of globally $C^{1}$ piecewise polynomials of degree $k+2$ with $k \geq 7$ that are $C^{4}$ at the vertices and $C^{2}$ at edges of the triangulation. A unisolvent set of degrees of freedom of this space is given locally by (cf. [32])

$$
\begin{aligned}
& D^{\alpha} z(a) \quad \forall a \in \mathcal{V}_{h}(T), \quad \forall|\alpha| \leq 4, \quad(140 \mathrm{DOFs}), \\
& \int_{e} z \sigma \quad \forall \sigma \in \mathcal{P}_{k-8}(e), \quad \forall e \in \mathcal{E}_{h}(T), \quad(6(k-7) \mathrm{DOFs}), \\
& \int_{e} \frac{\partial z}{\partial \boldsymbol{n}_{e_{ \pm}}} \sigma \quad \forall \sigma \in \mathcal{P}_{k-7}(e), \quad \forall e \in \mathcal{E}_{h}(T), \quad(12(k-6) \mathrm{DOFs}), \\
& \int_{e} \frac{\partial^{2} z}{\partial \boldsymbol{n}_{e_{ \pm}} \partial \boldsymbol{n}_{e_{ \pm}}} \sigma \quad \forall \sigma \in \mathcal{P}_{k-6}(e), \quad \forall e \in \mathcal{E}_{h}(T), \quad(18(k-5) \mathrm{DOFs}), \\
& \int_{F} z \sigma \quad \forall \sigma \in \mathcal{P}_{k-7}(F), \quad \forall F \in \mathcal{F}_{h}(T), \quad(2(k-5)(k-6) \mathrm{DOFs}), \\
& \int_{F} \frac{\partial z}{\partial \boldsymbol{n}_{F}} \sigma \quad \forall \sigma \in \mathcal{P}_{k-5}(F), \quad \forall F \in \mathcal{F}_{h}(T), \quad(2(k-3)(k-4) \mathrm{DOFs}), \\
& \int_{T}^{z \sigma} \quad \forall \sigma \in \mathcal{P}_{k-6}(T), \quad\left(\frac{1}{6}(k-3)(k-4)(k-5) \mathrm{DOFs}\right) .
\end{aligned}
$$

Here, $\boldsymbol{n}_{e_{ \pm}}$are two unit orthogonal normal vectors that are orthogonal to the edge $e$. In the case $k=7$, the set of DOFs listed in (3.1b) is omitted.

$\boldsymbol{H}^{1}$-conforming (velocity) finite element spaces. The $\boldsymbol{H}^{1}$-conforming finite element space, denoted by $\boldsymbol{V}_{h}$, consists of vector-valued, globally continuous piecewise polynomials of degree $k \geq 6$ that are $C^{2}$ on the vertices and $C^{1}$ on the edges of the triangulation. A unisolvent set of degrees of freedom of this space is given 

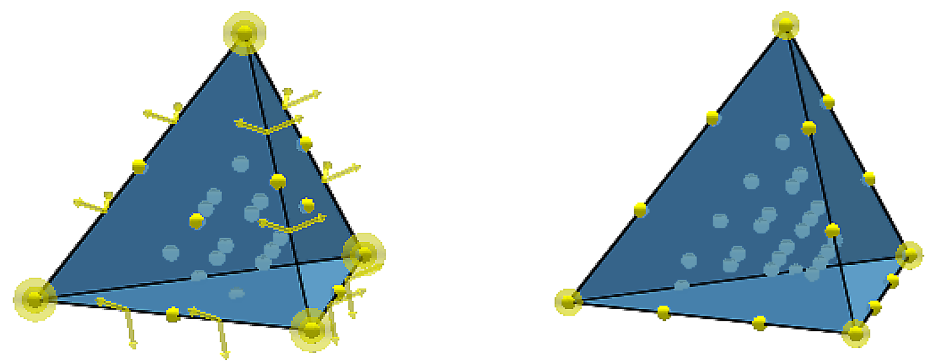

Figure 1. $\boldsymbol{H}^{1}(\Omega)$ finite element (left) and $L^{2}(\Omega)$ finite element (right) degrees of freedom with $k=6$.

by (cf. Figure 1)

$$
\begin{aligned}
& D^{\alpha} \boldsymbol{v}(a) \quad \forall|\alpha| \leq 2, \quad \forall a \in \mathcal{V}_{h}(T), \quad(120 \text { DOFs }), \\
& \int_{e} \boldsymbol{v} \cdot \boldsymbol{\kappa} \quad \forall \boldsymbol{\kappa} \in \mathcal{P}_{k-6}(e), \quad \forall e \in \mathcal{E}_{h}(T), \quad(18(k-5) \text { DOFs }), \\
& \int_{e} \frac{\partial \boldsymbol{v}}{\partial \boldsymbol{n}_{e_{ \pm}}} \cdot \boldsymbol{\sigma} \quad \forall \boldsymbol{\sigma} \in \mathcal{P}_{k-5}(e), \quad \forall e \in \mathcal{E}_{h}(T), \quad(36(k-4) \mathrm{DOFs}), \\
& \int_{F} \boldsymbol{v} \cdot \boldsymbol{\psi} \quad \forall \boldsymbol{\psi} \in \mathcal{P}_{k-6}(F), \quad \forall F \in \mathcal{F}_{h}(T), \quad(6(k-4)(k-5) \text { DOFs }), \\
& \int_{T} \boldsymbol{v} \cdot \boldsymbol{\varphi} \quad \forall \boldsymbol{\varphi} \in \mathcal{P}_{k-4}(T), \quad\left(\frac{1}{2}(k-1)(k-2)(k-3) \text { DOFs }\right) .
\end{aligned}
$$

It is simple to see that these degrees of freedom uniquely determine a function in $\boldsymbol{V}_{h}$ on each tetrehedron $T \in \mathcal{T}_{h}$. We sketch the main argument.

First note that there are $\frac{1}{2}(k+3)(k+2)(k+1)$ degrees of freedom given in (3.2), which is exactly the dimension of $\mathcal{P}_{k}(T)$. Therefore to show unisolvency, it suffices to show that if $\boldsymbol{v} \in \mathcal{P}_{k}(T)$ vanishes at the degrees of freedom (3.2), then $\boldsymbol{v}$ is identically zero. In this case, it is clear that $\boldsymbol{v}$ and $D \boldsymbol{v}$ vanish on all edges. Therefore, we may write $\left.\boldsymbol{v}\right|_{F}=b_{F}^{2} p_{F}$ for some $p_{F} \in \mathcal{P}_{k-6}(T)$ for all $F \in \mathcal{F}_{h}(T)$. It then follows from the fourth set of DOFs, that $\left.\boldsymbol{v}\right|_{\partial T}=0$ (this also shows the conforming property $\boldsymbol{V}_{h} \subset \boldsymbol{H}^{1}(\Omega)$ ). Therefore $\boldsymbol{v}=b_{T} \boldsymbol{p}$ for some $\boldsymbol{p} \in \mathcal{P}_{k-4}(T)$. By the last set of DOFs, we deduce $\boldsymbol{v} \equiv 0$. Thus, the DOFs listed in (3.2) form a unisolvent set.

Remark 3.1. The degrees of freedom (3.2) with $k=5$ still form a unisolvent set for the space $\mathcal{P}_{5}(T)$. However, we require the degrees of freedom (3.2d) in the proof of Lemma 4.5 below; hence, the restriction $k \geq 6$.

$L^{2}$-conforming (pressure) finite element spaces. Next, we set $Q_{h}$ to be the space of piecewise polynomials of degree $k-1$ that are $C^{1}$ on the vertices and $C^{0}$ on the edges of the triangulation. It is easy to see that such functions are uniquely determined by the following values:

$$
\begin{aligned}
& D^{\alpha} q(a) \quad \forall|\alpha| \leq 1, \forall a \in \mathcal{V}_{h}(T), \quad \int_{e} q \xi \quad \forall \xi \in \mathcal{P}_{k-5}(e), \forall e \in \mathcal{E}_{h}(T), \\
& \int_{T} q \zeta \quad \forall \zeta \in \mathcal{P}_{k-1}(T) \text { such that }\left.\zeta\right|_{e}=0, \forall e \in \mathcal{E}_{h}(T) .
\end{aligned}
$$


$\boldsymbol{H}^{1}(\operatorname{curl} ; \Omega)$-conforming finite element spaces. Finally, we consider the construction of the $\boldsymbol{H}^{1}(\mathbf{c u r l} ; \Omega)$-conforming finite element space $\boldsymbol{\Upsilon}_{h}$ in the sequence (1.3). We can infer properties of $\boldsymbol{\Upsilon}_{h}$ from those of the already constructed spaces. For example, since we wish the inclusion $\operatorname{grad} \Sigma_{h} \subset \boldsymbol{\Upsilon}_{h}$ to hold, we expect $\boldsymbol{\Upsilon}_{h}$ to consist of functions that are $C^{3}$ at vertices and $C^{1}$ at edges of the triangulation. Moreover, if curl $\boldsymbol{\Upsilon}_{h} \subset \boldsymbol{V}_{h}$ then the curls of functions in $\boldsymbol{\Upsilon}_{h}$ will be $C^{1}$ on edges of the triangulation.

The number of degrees of freedom for each vertex, edge and face can also be deduced by (1.3) and a counting argument. The degrees of freedom of $\Sigma_{h}$ given in (3.1) show that the (global) dimension of $\Sigma_{h}$ is $35 \mathbb{V}+(6 k-34) \mathbb{E}+\left(k^{2}-9 k+21\right) \mathbb{F}+$ $\frac{1}{6}(k-3)(k-4)(k-5) \mathbb{T}$, where $\mathbb{V}, \mathbb{E}, \mathbb{F}$ and $\mathbb{T}$ denote, respectively, the number of vertices, edges, faces and tetrahedra in the triangulation. Likewise the degrees of freedom (3.2) and (3.3) give us $\operatorname{dim} \boldsymbol{V}_{h}=30 \mathbb{V}+(9 k-39) \mathbb{E}+\frac{3}{2}(k-4)(k-5) \mathbb{F}+$ $\frac{1}{2}(k-1)(k-2)(k-3) \mathbb{T}$, and $\operatorname{dim} Q_{h}=4 \mathbb{V}+(k-4) \mathbb{E}+\left(\frac{1}{6} k^{3}+\frac{1}{2} k^{2}-\frac{17}{3} k+8\right) \mathbb{T}$. In order for the sequence (1.3) to be exact, the global dimension of $\boldsymbol{\Upsilon}_{h}$ must equal:

$$
\begin{aligned}
\operatorname{dim} \boldsymbol{\Upsilon}_{h}=\operatorname{dim} \boldsymbol{V}_{h}+\operatorname{dim} \Sigma_{h}-\operatorname{dim} Q_{h}-1 \\
=61 \mathbb{V}+(14 k-69) \mathbb{E}+\left(\frac{5}{2} k^{2}-\frac{45}{2} k+51\right) \mathbb{F}+\left(\frac{1}{2} k^{3}-\frac{11}{2} k^{2}+19 k-21\right) \mathbb{T}-1 .
\end{aligned}
$$

Using the relation $\mathbb{V}+\mathbb{F}-\mathbb{T}-\mathbb{E}=1$ (cf. [18, Theorem 16.14]), we find

$$
\operatorname{dim} \Upsilon_{h}=60 \mathbb{V}+(14 k-68) \mathbb{E}+\frac{5}{2}(k-4)(k-5) \mathbb{F}+\frac{1}{2}(k-2)(k-5)(k-4) \mathbb{T} .
$$

Furthermore, we see that the dimension of the local space, denoted by $\mathbf{\Upsilon}(T)$, will be

$$
\begin{aligned}
\operatorname{dim} \Upsilon(T) & =60(4)+(14 k-68)(6)+\frac{5}{2}(k-4)(k-5)(4)+\frac{1}{2}(k-2)(k-5)(k-4) \\
& =\frac{1}{2}(k+4)(k+3)(k+2)=\operatorname{dim} \mathcal{P}_{k+1}(T) .
\end{aligned}
$$

We now precisely state the $\boldsymbol{H}^{1}(\mathbf{c u r l} ; \Omega)$-conforming finite element space $\mathbf{\Upsilon}_{h}$. This space consists of vector-valued piecewise polynomials of degree $k+1(k \geq 6)$ that are $C^{3}$ at vertices of the triangulation, $C^{1}$ at edges in the triangulation and whose curls are $C^{1}$ at edges in the triangulation. The first set of degrees of freedom which determine a function $\boldsymbol{z} \in \boldsymbol{\Upsilon}_{h}$ is given locally by

$$
\begin{aligned}
& D^{\alpha} \boldsymbol{z}(a) \quad \forall|\alpha| \leq 3, \quad \forall a \in \mathcal{V}_{h}(T), \quad(240 \text { DOFs }), \\
& \int_{e} \boldsymbol{z} \cdot \boldsymbol{\sigma} \quad \forall \boldsymbol{\sigma} \in \mathcal{P}_{k-7}(e), \quad \forall e \in \mathcal{E}_{h}(T), \quad(18(k-6) \mathrm{DOFs}) \\
& \int_{e} \frac{\partial \boldsymbol{z}}{\partial \boldsymbol{n}_{e_{ \pm}}} \cdot \boldsymbol{\mu} \quad \forall \boldsymbol{\mu} \in \mathcal{P}_{k-6}(e), \quad \forall e \in \mathcal{E}_{h}(T), \quad(36(k-5) \text { DOFs }), \\
& \int_{F}\left(\boldsymbol{z} \cdot \boldsymbol{n}_{F}\right) \varphi \quad \forall \varphi \in \mathcal{P}_{k-5}(F), \quad \forall F \in \mathcal{F}_{h}(T), \quad(2(k-3)(k-4) \text { DOFs }), \\
& \int_{F}\left(\boldsymbol{z} \times \boldsymbol{n}_{F}\right) \cdot \boldsymbol{\rho} \quad \forall \boldsymbol{\rho} \in \boldsymbol{D}_{k-6}(F), \\
& \forall F \in \mathcal{F}_{h}(T), \quad(4(k-4)(k-6) \text { DOFs }),
\end{aligned}
$$




$$
\begin{aligned}
\int_{F}\left(\operatorname{curl} \boldsymbol{z} \times \boldsymbol{n}_{F}\right) \cdot \boldsymbol{\psi} \quad \forall \boldsymbol{\psi} & \in \mathcal{P}_{k-6}(F), \\
\forall F \in \mathcal{F}_{h}(T), \quad(4(k-4)(k-5) \text { DOFs }), & \left(\frac{1}{2}(k-2)(k-4)(k-5) \text { DOFs }\right),
\end{aligned}
$$

where $\boldsymbol{D}_{m}(T)=\mathcal{P}_{m-1}(T)+\boldsymbol{x} \mathcal{P}_{m-1}(T)$ is the (local) Nedelec space [22. In the case $k=6$, the degrees of freedom (3.4b) and (3.4e) are omitted.

The description of the remaining DOFs require additional notation. Let $\boldsymbol{t}_{e}$ be a unit vector, tangent to the edge $e$, and let $\boldsymbol{n}_{e_{ \pm}}$be unit vectors chosen such that $\left\{\boldsymbol{t}_{e}, \boldsymbol{n}_{e_{ \pm}}\right\}$is an orthonormal basis of $\mathbb{R}^{3}$. We assume, without loss of generality, that the unitary matrix $B_{e}:=\left(\boldsymbol{t}_{e} \boldsymbol{n}_{e_{+}} \boldsymbol{n}_{e_{-}}\right) \in \mathbb{R}^{3 \times 3}$ satisfies $\operatorname{det}\left(B_{e}\right)=1$. The additional degrees of freedom are then given by

$$
\begin{gathered}
\int_{e} \frac{\partial(\operatorname{curl} \boldsymbol{z})}{\partial \boldsymbol{n}_{e_{+}}} \cdot \boldsymbol{\kappa} \quad \forall \boldsymbol{\kappa} \in \mathcal{P}_{k-5}(e), \quad \forall e \in \mathcal{E}_{h}(T), \quad(18(k-4) \text { DOFs }), \\
\int_{e}\left(I_{3 \times 3}-\boldsymbol{n}_{e_{-}} \boldsymbol{n}_{e_{-}}^{t}\right) \frac{\partial(\operatorname{curl} \boldsymbol{z})}{\partial \boldsymbol{n}_{e_{-}}} \cdot \boldsymbol{\sigma} \quad \forall \boldsymbol{\sigma} \in \mathcal{P}_{k-5}(e), \\
\forall e \in \mathcal{E}_{h}(T), \quad(12(k-4) \text { DOFs }) .
\end{gathered}
$$

Notice that the number of degrees of freedom listed in (3.4) is exactly the dimension of $\mathcal{P}_{k+1}(T)$. The standard unisolvency proof then proceeds by showing that if $\boldsymbol{z}$ vanishes at the values listed in (3.4), then $\boldsymbol{z} \equiv 0$. We break up this argument into several steps. First, we establish the following result.

Lemma 3.2. Suppose $\boldsymbol{z} \in \mathcal{P}_{k+1}(T)$ vanishes at the degrees of freedom (3.4a)(3.4C), (3.4h) -(3.4i). Then $\boldsymbol{z}, D \boldsymbol{z}$ and $D(\operatorname{curl} \boldsymbol{z})$ vanish on all edges of $T$.

Proof. The assertion $\left.\boldsymbol{z}\right|_{e}=0$ and $\left.D \boldsymbol{z}\right|_{e}=0$ trivially follows from (3.4a)-(3.4c). We also see from (3.4h) - (3.4i) that $\partial(\operatorname{curl} \boldsymbol{z}) / \partial \boldsymbol{n}_{e_{+}}=0, \boldsymbol{t}_{e} \cdot \partial(\operatorname{curl} \boldsymbol{z}) / \partial \boldsymbol{n}_{e_{-}}=0$ and $\boldsymbol{n}_{e_{+}} \cdot \partial(\operatorname{curl} \boldsymbol{z}) / \partial \boldsymbol{n}_{e_{-}}=0$ on all edges. Furthermore, by Lemma A.2 we have

$$
\boldsymbol{n}_{e_{-}} \cdot \frac{\partial(\operatorname{curl} \boldsymbol{z})}{\partial \boldsymbol{n}_{e_{-}}}=-\boldsymbol{t}_{e} \cdot \frac{\partial(\operatorname{curl} \boldsymbol{z})}{\partial \boldsymbol{t}_{e}}-\boldsymbol{n}_{e_{+}} \cdot \frac{\partial(\operatorname{curl} \boldsymbol{z})}{\partial \boldsymbol{n}_{e_{+}}}=0 .
$$

Therefore, $\left.D(\operatorname{curl} \boldsymbol{z})\right|_{e}=0$.

Lemma 3.3. Suppose $\boldsymbol{z} \in \mathcal{P}_{k+1}(T)$ vanishes at the degrees of freedom (3.4a)(3.4f), (3.4h) - (3.4i) restricted to a single face $F \subset \partial T$. Then $\boldsymbol{z}=\operatorname{curl} \boldsymbol{z}=0$ on $F$.

Remark 3.4. This result establishes the conforming property $\mathbf{\Upsilon}_{h} \subset \boldsymbol{H}^{1}(\operatorname{curl} ; \Omega)$.

Proof. By Lemma $3.2, \boldsymbol{z}, D \boldsymbol{z}$ and $D(\operatorname{curl} \boldsymbol{z})$ all vanish on $\partial F$. It then follows from (3.4d) that $\left.\boldsymbol{z} \cdot \boldsymbol{n}\right|_{F}=0$. Furthermore, we may write $\left.\operatorname{curl} \boldsymbol{z}\right|_{F}=b_{F}^{2} \boldsymbol{p}$ for some $\boldsymbol{p} \in \mathcal{P}_{k-6}(F)$. Therefore by (3.4f), $\operatorname{curl} \boldsymbol{z} \times\left.\boldsymbol{n}\right|_{F}=0$.

Next, Stokes Theorem gives

$$
\int_{F}\left(\boldsymbol{z}_{F} \cdot \operatorname{curl}_{F} q-\left(\operatorname{curl}_{F} \boldsymbol{z}_{F}\right) q\right)=\int_{\partial F} \boldsymbol{z} \cdot \boldsymbol{t} q=0 .
$$

Consequently, by (3.4e) we have

$$
\int_{F}\left(\operatorname{curl}_{F} \boldsymbol{z}_{F}\right) q=0 \quad \forall q \in \mathcal{P}_{k-6}(F) .
$$


With the correct orientation, we have $\operatorname{curl}_{F} \boldsymbol{z}_{F}=(\operatorname{curl} \boldsymbol{z}) \cdot \boldsymbol{n}_{F}=b_{F}^{2} \boldsymbol{p} \cdot \boldsymbol{n}_{F}$. Since $\boldsymbol{p} \in \mathcal{P}_{k-6}(F)$, we deduce from (3.6) that $\boldsymbol{p} \cdot \boldsymbol{n}_{F}=0$ and therefore $(\operatorname{curl} \boldsymbol{z}) \cdot \boldsymbol{n}_{F}=0$. Hence, $\operatorname{curl} \boldsymbol{z}$ vanishes on $\partial T$. Moreover, $\operatorname{curl}_{F} \boldsymbol{z}_{F}=0$ implies $\boldsymbol{z}_{F}=\operatorname{grad}_{F} \phi$ for some $\phi \in \mathcal{P}_{k+2}(F)$. Since $\boldsymbol{z}$ and $D \boldsymbol{z}$ vanish on $\partial F$, we may assume that $\phi$ vanishes up to second order on $\partial F$. Consequently, $\phi=b_{F}^{3} r$ for some $r \in \mathcal{P}_{k-7}(F)$. We then have

$$
0=\int_{F} \boldsymbol{z}_{F} \cdot \boldsymbol{q}=-\int_{F} b_{F}^{3} r \operatorname{div}_{F} \boldsymbol{q} \quad \forall \boldsymbol{q} \in \boldsymbol{D}_{k-6}(F) .
$$

Since the image of $\boldsymbol{D}_{k-6}(F)$ under the divergence operator is $\mathcal{P}_{k-7}(F)$ [22], we deduce that $r \equiv 0$ and therefore $\boldsymbol{z}_{F}=0$. Since $\boldsymbol{z} \cdot \boldsymbol{n}$ vanishes on $F,\left.\boldsymbol{z}\right|_{F}=0$.

Theorem 3.5. The degrees of freedom (3.4) are unisolvent on $\boldsymbol{\Upsilon}_{h}$.

Proof. Suppose $\boldsymbol{z} \in \mathcal{P}_{k+1}(T)$ vanishes at the values listed in (3.4). It suffices to show $z \equiv 0$.

By Lemma 3.3 we have $\boldsymbol{z} \in \boldsymbol{H}_{0}^{1}(\mathbf{c u r l} ; T)$ and

$$
\int_{T} \boldsymbol{z} \cdot \boldsymbol{\rho}=0 \quad \forall \boldsymbol{\rho} \in \boldsymbol{D}_{k-5}(T) .
$$

Denote by $\hat{T}$ the reference tetrahedron with vertices $(0,0,0),(1,0,0),(0,1,0)$ and $(0,0,1)$. Let $\boldsymbol{F}_{T}: \hat{T} \rightarrow T$ with $\boldsymbol{F}_{T}(\hat{\boldsymbol{x}})=A \hat{\boldsymbol{x}}+\boldsymbol{b}\left(A \in \mathbb{R}^{3 \times 3}, \boldsymbol{b} \in \mathbb{R}^{3}\right)$ be an affine transformation, mapping the reference tetrahedron onto $T$. For given $\boldsymbol{z} \in$ $\mathcal{P}_{k+1}(T) \cap \boldsymbol{H}_{0}^{1}(\operatorname{curl} ; T)$, define the transformation $\boldsymbol{z}(\boldsymbol{x})=A^{-T} \hat{\boldsymbol{z}}(\hat{\boldsymbol{x}})$ with $\boldsymbol{x}=$ $\boldsymbol{F}_{T}(\hat{\boldsymbol{x}})$. We then have $\operatorname{curl} \boldsymbol{z}=A(\widehat{\operatorname{curl}} \hat{\boldsymbol{z}}) / \operatorname{det}(A)$, where $\widehat{\operatorname{curl}}$ denotes the curl operator with respect to the $\hat{\boldsymbol{x}}$-variables; see [21] for details. Consequently, $\hat{\boldsymbol{z}} \in$ $\mathcal{P}_{k+1}(\hat{T}) \cap \boldsymbol{H}_{0}^{1}(\operatorname{curl} ; \hat{T})$ and by (3.7),

$$
\int_{\hat{T}}(\widehat{\operatorname{curl}} \hat{\boldsymbol{z}}) \cdot \hat{\boldsymbol{\rho}}=\int_{T}(\operatorname{curl} \boldsymbol{z}) \cdot \boldsymbol{\rho}=\int_{T} \boldsymbol{z} \cdot(\operatorname{curl} \boldsymbol{\rho})=0 \quad \forall \boldsymbol{\rho} \in \mathcal{P}_{k-5}(T),
$$

where $\hat{\boldsymbol{\rho}} \in \boldsymbol{P}_{k-5}(\hat{T})$ satisfies $\hat{\boldsymbol{\rho}}(\hat{\boldsymbol{x}})=\boldsymbol{\rho}(\boldsymbol{x})$ with $\boldsymbol{x}=\boldsymbol{F}_{T}(\hat{\boldsymbol{x}})$. Next, since $\widehat{\operatorname{curl}} \hat{\boldsymbol{z}} \times \hat{\boldsymbol{n}}$ and $\hat{\boldsymbol{z}}$ both vanish on $\partial \hat{T}$, then $\widehat{\operatorname{curl}} \hat{\boldsymbol{z}}$ vanishes on the boundary of $\hat{T}$ as well. Therefore we may write $\widehat{\operatorname{curl}} \hat{\boldsymbol{z}}=b_{\hat{T}} \hat{\boldsymbol{q}}$ for some $\hat{\boldsymbol{q}} \in \mathcal{P}_{k-4}(\hat{T})$. Restricting the identity

$$
0=\widehat{\operatorname{div}}\left(b_{\hat{T}} \hat{\boldsymbol{q}}\right)=b_{\hat{T}} \widehat{\operatorname{div}} \hat{\boldsymbol{q}}+\widehat{\operatorname{grad}} b_{\hat{T}} \cdot \hat{\boldsymbol{q}}
$$

to an arbitrary face $\hat{F} \subset \partial \hat{T}$, we deduce $\left.\widehat{\operatorname{grad}} b_{\hat{T}} \cdot \hat{\boldsymbol{q}}\right|_{\hat{F}}=0$. By (2.1), $\widehat{\operatorname{grad}} b_{\hat{T}}=$ $b_{\hat{F}} \widehat{\operatorname{grad}} \lambda_{\hat{F}}$ on $F$, and $\operatorname{grad} \lambda_{\hat{F}}$ is parallel to the normal $\boldsymbol{n}_{\hat{F}}$. Since the face bubble $b_{F}$ is strictly positive on $F$ we have $\hat{\boldsymbol{q}} \cdot \hat{\boldsymbol{n}}=0$ on $\partial \hat{T}$. Therefore we may write $\hat{q}_{j}=\hat{x}_{j} \hat{\psi}_{j}$ for some $\hat{\boldsymbol{\psi}} \in \boldsymbol{P}_{k-5}(\hat{T})$. Setting $\boldsymbol{\rho}=\boldsymbol{\psi}$ in (3.8) we deduce that $\hat{\boldsymbol{q}} \equiv 0$, and therefore $\operatorname{curl} \boldsymbol{z} \equiv 0$.

Since $\boldsymbol{z}$ is curl-free, we may write $\boldsymbol{z}=\operatorname{grad} p$ for some $p \in \mathcal{P}_{k+2}(T) \cap H_{0}^{2}(T)$. By (3.7) and integrating by parts we obtain

$$
0=\int_{T} \boldsymbol{z} \cdot \boldsymbol{\rho}=-\int_{T} p(\operatorname{div} \boldsymbol{\rho})=0 \quad \forall \boldsymbol{\rho} \in \boldsymbol{D}_{k-5}(T) .
$$

Since the divergence operator is surjective from $\boldsymbol{D}_{k-5}(T)$ to $\mathcal{P}_{k-6}(T)$ 22], there holds $\int_{T} p r=0$ for all $r \in \mathcal{P}_{k-6}(T)$. Since $p \in \mathcal{P}_{k+2}(T) \cap H_{0}^{2}(T)$, this implies $p \equiv 0$, and therefore $\boldsymbol{z} \equiv 0$. 


\section{The Discrete De Rham COMPleX}

In light of the definitions of the finite element spaces, the inclusions grad $\Sigma_{h} \subset$ $\boldsymbol{\Upsilon}_{h}$, curl $\boldsymbol{\Upsilon}_{h} \subset \boldsymbol{V}_{h}$ and $\operatorname{div} \boldsymbol{V}_{h} \subset Q_{h}$ hold. Therefore the sequence (1.3) is a complex. This section is devoted to showing that the complex is exact. In addition, we derive some useful stability properties.

4.1. A local smooth de Rham complex. We first focus our attention to a local de Rham complex consisting of polynomial spaces. For an element $T \in \mathcal{T}_{h}$ and positive integer $k$, define the spaces $\Sigma_{k+2,0}(T)=\mathcal{P}_{k+2}(T) \cap H_{0}^{2}(\Omega), \boldsymbol{\Upsilon}_{k+1,0}(T):=$ $\mathcal{P}_{k+1}(T) \cap \boldsymbol{H}_{0}^{1}(\operatorname{curl} ; T), \boldsymbol{V}_{k, 0}:=\mathcal{P}_{k}(T) \cap \boldsymbol{H}_{0}^{1}(T)$ and

$$
Q_{k-1,0}(T):=\left\{q \in \mathcal{P}_{k-1}(T):\left.q\right|_{e}=0, \int_{T} q=0\right\} .
$$

In this section, we consider the local sequence given by

$$
0 \longrightarrow \Sigma_{k+2,0}(T) \stackrel{\text { grad }}{\longrightarrow} \Upsilon_{k+1,0}(T) \stackrel{\text { curl }}{\longrightarrow} \boldsymbol{V}_{k, 0}(T) \stackrel{\text { div }}{\longrightarrow} Q_{k-1,0}(T) \longrightarrow 0 .
$$

It is clear from the definitions of the spaces that (4.2) is a complex. In fact we show that the complex (4.2) is exact.

Using the definitions of the spaces $\mathbf{\Upsilon}_{k+1,0}(T)$ and $\Sigma_{k+2,0}(T)$, it is easy to see that if $\boldsymbol{z} \in \boldsymbol{\Upsilon}_{k+1,0}(T)$ satisfies $\operatorname{curl} \boldsymbol{z}=0$, then $\boldsymbol{z}=\operatorname{grad} w$ for some $w \in \Sigma_{k+2,0}(T)$. Thus, to prove that (4.2) is exact we must show that (i) if $\boldsymbol{v} \in \boldsymbol{V}_{k, 0}(T)$ is solenoidal, then $\boldsymbol{v}=\operatorname{curl} \boldsymbol{z}$ for some $\boldsymbol{z} \in \mathbf{\Upsilon}_{k+1,0}(T)$ and (ii) the divergence operator div : $\boldsymbol{V}_{k, 0}(T) \rightarrow Q_{k-1,0}(T)$ is surjective. We address the first issue in the next lemma.

Lemma 4.1. Suppose $\boldsymbol{v} \in \boldsymbol{V}_{k, 0}(T)$ satisfies $\operatorname{div} \boldsymbol{v}=0$. Then there exists a function $\boldsymbol{z} \in \mathbf{\Upsilon}_{k+1,0}(T)$ such that $\boldsymbol{v}=\operatorname{curl} \boldsymbol{z}$.

Proof. By Lemma 2.5 there exists $\boldsymbol{w} \in \boldsymbol{H}_{0}^{1}(\operatorname{curl} ; T)$ satisfying $\operatorname{curl} \boldsymbol{w}=\boldsymbol{v}$. Let $\boldsymbol{z} \in \mathcal{P}_{k+1}(T)$ be the unique function that vanishes at the the degrees of freedom (3.4a) - (3.4f), (3.4h)- (3.4i) and satisfies

$$
\int_{T} \boldsymbol{z} \cdot \boldsymbol{\rho}=\int_{T} \boldsymbol{w} \cdot \boldsymbol{\rho} \quad \forall \boldsymbol{\rho} \in \boldsymbol{D}_{k-5}(T) .
$$

By Lemma 3.3, there holds $\boldsymbol{z} \in \boldsymbol{\Upsilon}_{k+1,0}(T)$. Moreover, integration by parts gives us

$$
\int_{T} \operatorname{curl} z \cdot \rho=\int_{T} z \cdot \operatorname{curl} \rho=\int_{T} w \cdot \operatorname{curl} \rho=\int_{T} \operatorname{curl} w \cdot \rho=\int_{T} \boldsymbol{v} \cdot \boldsymbol{\rho}
$$

for all $\boldsymbol{\rho} \in \mathcal{P}_{k-5}(T)$.

Since $\operatorname{curl} \boldsymbol{z}$ vanishes on $\partial T$, there exists $\boldsymbol{q} \in \mathcal{P}_{k-4}(T)$ such that $\operatorname{curl} \boldsymbol{z}=b_{T} \boldsymbol{q}$. By Theorem 3.5, the function $\boldsymbol{q}$ satisfies $\boldsymbol{q} \cdot \boldsymbol{n}=0$ on $\partial T$. By similar arguments, there holds $\boldsymbol{v}=b_{T} \boldsymbol{p}$ with $\boldsymbol{p} \in \mathcal{P}_{k-4}(T)$ and $\left.\boldsymbol{p} \cdot \boldsymbol{n}\right|_{\partial T}=0$. Making a change of variables to the reference tetrahedron, we have $\hat{q}_{j}=\hat{x}_{j} \hat{\psi}_{j}$ and $\hat{p}_{j}=\hat{x}_{j} \hat{\varphi}_{j}$ for some $\hat{\boldsymbol{\psi}}, \hat{\boldsymbol{\varphi}} \in \mathcal{P}_{k-5}(\hat{T})$. By (4.3) we have $\hat{\boldsymbol{\psi}}=\hat{\boldsymbol{\varphi}}$, and therefore $\operatorname{curl} \boldsymbol{z}=\boldsymbol{v}$.

Theorem 4.2. The complex (4.2) is exact.

Proof. In light of the previous discussion, it suffices to show div : $\boldsymbol{V}_{k, 0}(T) \rightarrow$ $Q_{k-1,0}(T)$ is surjective to complete the proof. This is achieved by a simple counting argument. We assume that $k \geq 4$, as the result is trivial in the case $k \leq 3$. 
First we note that

$$
\begin{aligned}
\operatorname{dim}\left(\operatorname{curl} \boldsymbol{\Upsilon}_{k+1,0}(T)\right) & =\operatorname{dim} \boldsymbol{\Upsilon}_{k+1,0}(T)-\operatorname{dim}\left(\operatorname{grad} \Sigma_{k+2,0}(T)\right) \\
& =\operatorname{dim} \boldsymbol{\Upsilon}_{k+1,0}(T)-\operatorname{dim} \mathcal{P}_{k-6}(T)
\end{aligned}
$$

Therefore by Lemma 4.1 we have

$$
\begin{aligned}
\operatorname{dim}\left(\operatorname{div} \boldsymbol{V}_{k, 0}\right) & =\operatorname{dim} \boldsymbol{V}_{k, 0}-\operatorname{dim}\left(\operatorname{curl} \boldsymbol{\Upsilon}_{k+1,0}\right) \\
& =\operatorname{dim} \boldsymbol{P}_{k-4}(T)+\operatorname{dim} \mathcal{P}_{k-6}(T)-\operatorname{dim} \boldsymbol{\Upsilon}_{k+1,0}(T) .
\end{aligned}
$$

By Lemma 3.3 and Theorem 3.5. we have $\operatorname{dim} \boldsymbol{\Upsilon}_{k+1,0}(T) \leq \operatorname{dim} \boldsymbol{D}_{k-5}(T)$. Therefore,

$$
\begin{aligned}
& \operatorname{dim}\left(\operatorname{div} \boldsymbol{V}_{k, 0}\right) \geq \operatorname{dim} \mathcal{P}_{k-4}(T)+\operatorname{dim} \mathcal{P}_{k-6}(T)-\operatorname{dim} \boldsymbol{D}_{k-5}(T) \\
& =\frac{1}{2}(k-1)(k-2)(k-3)+\frac{1}{6}(k-3)(k-4)(k-5)-\frac{1}{2}(k-2)(k-4)(k-5) \\
& =\frac{1}{6} k^{3}+\frac{1}{2} k^{2}-\frac{17}{3} k+7 .
\end{aligned}
$$

Next, it is easy to see that $6 k-7$ linearly independent constraints are imposed in the space $Q_{k-1,0}(T)$. Thus,

$$
\operatorname{dim} Q_{k-1,0}(T)=\operatorname{dim} \mathcal{P}_{k-1}(T)-6 k+7=\frac{1}{6} k^{3}+\frac{1}{2} k^{2}-\frac{17}{3} k+7 .
$$

Therefore, $\operatorname{dim}\left(\operatorname{div} \boldsymbol{V}_{k, 0}\right) \geq \operatorname{dim} Q_{k-1,0}(T)$. But since $\operatorname{div} \boldsymbol{V}_{k, 0} \subseteq Q_{k-1,0}(T)$, we must have $\operatorname{div} \boldsymbol{V}_{k, 0}=Q_{k-1,0}(T)$, i.e., div $: \boldsymbol{V}_{k, 0}(T) \rightarrow Q_{k-1,0}(T)$ is surjective.

Remark 4.3. For the two-dimensional version of Theorem 4.2. see [23, Proposition 3.1] and [27, Lemma 2.5].

Remark 4.4. Combining Theorem 4.2 with a simple scaling argument, we conclude that for any $q \in Q_{k-1,0}(T)$, there exists $\boldsymbol{v} \in \boldsymbol{V}_{k, 0}(T)$ such that $\operatorname{div} \boldsymbol{v}=q$ and $\|\boldsymbol{v}\|_{H^{1}(T)} \leq C\|q\|_{L^{2}(T)}$.

4.2. The global smooth discrete de Rham complex. We now turn our attention to the (global) discrete complex given in (1.3). Again, it is clear that if $k \geq 7$ and $\boldsymbol{z} \in \mathbf{\Upsilon}_{h}$ satisfies $\operatorname{curl} \boldsymbol{z}=0$, then there exists $w \in \Sigma_{h}$, unique up to a constant, such that $\boldsymbol{z}=\operatorname{grad} w$. The next lemma shows that the divergence operator div : $\boldsymbol{V}_{h} \rightarrow Q_{h}$ is surjective.

Lemma 4.5. For every $q \in Q_{h}$, there exists $\boldsymbol{v} \in \boldsymbol{V}_{h}$ with $\operatorname{div} \boldsymbol{v}=q$ and $\|\boldsymbol{v}\|_{H^{1}(\Omega)} \leq$ $C\|q\|_{L^{2}(\Omega)}$.

Proof. Given $q \in Q_{h}$, let $\boldsymbol{w} \in \boldsymbol{H}^{1}(\Omega)$ satisfy $\operatorname{div} \boldsymbol{w}=q$ and $\|\boldsymbol{w}\|_{H^{1}(\Omega)} \leq C\|q\|_{L^{2}(\Omega)}$ [16. We also let $\boldsymbol{I}_{h} \boldsymbol{w} \in \mathcal{P}_{k}$ denote the Scott-Zhang interpolant of $\boldsymbol{w}$, where $\boldsymbol{P}_{k}=$ $\left[\mathcal{P}_{k}\right]^{3}$ is the vector-valued Lagrange finite element space of degree $k$ (cf. Lemma 2.3). We then prescribe $\boldsymbol{v}_{1}=\left(v_{1,1}, v_{1,2}, v_{1,3}\right)^{t} \in \boldsymbol{V}_{h}$ by the following criterion:

(i) $\boldsymbol{v}_{1}(a)=\boldsymbol{I}_{h} \boldsymbol{w}(a), \quad \frac{D^{\alpha}\left(\partial v_{1, i}\right)}{\partial x_{i}}(a)=\frac{1}{3} D^{\alpha} q(a)$, $\frac{D^{\alpha}\left(\partial v_{1, i}\right)}{\partial x_{j}}(a)=0(i \neq j)$

for all multi-indices $|\alpha| \leq 1$ and for all vertices $a \in \mathcal{V}_{h}$; 
(ii) $\int_{e} \boldsymbol{v}_{1} \cdot \boldsymbol{\kappa}=\int_{e} \boldsymbol{I}_{h} \boldsymbol{w} \cdot \boldsymbol{\kappa}$ for all $\boldsymbol{\kappa} \in \mathcal{P}_{k-6}(e)$ and edges $e \in \mathcal{E}_{h}$.

We note that $\frac{\partial \boldsymbol{v}_{1}}{\partial \boldsymbol{t}_{e}}$ on each edge is uniquely determined by conditions (i)-(ii);

(iii) $\int_{e} \frac{\partial \boldsymbol{v}_{1}}{\partial \boldsymbol{n}_{e_{+}}} \cdot \boldsymbol{n}_{e_{+}} \sigma=\frac{1}{2} \int_{e}\left(q-\frac{\partial \boldsymbol{v}_{1}}{\partial \boldsymbol{t}_{e}} \cdot \boldsymbol{t}_{e}\right) \sigma$,

$\int_{e} \frac{\partial \boldsymbol{v}_{1}}{\partial \boldsymbol{n}_{e_{-}}} \cdot \boldsymbol{n}_{e_{-}} \sigma=\frac{1}{2} \int_{e}\left(q-\frac{\partial \boldsymbol{v}_{1}}{\partial \boldsymbol{t}_{e}} \cdot \boldsymbol{t}_{e}\right) \sigma$,

$\int_{e} \frac{\partial \boldsymbol{v}_{1}}{\partial \boldsymbol{n}_{e_{+}}} \cdot \boldsymbol{n}_{e_{-}} \sigma=\frac{\partial \boldsymbol{v}_{1}}{\partial \boldsymbol{n}_{e_{-}}} \cdot \boldsymbol{n}_{e_{+}} \sigma=\int_{e} \frac{\partial \boldsymbol{v}_{1}}{\partial \boldsymbol{n}_{e_{ \pm}}} \cdot \boldsymbol{t}_{e} \sigma=0$,

for all $\sigma \in \mathcal{P}_{k-5}(e)$ and edges $e \in \mathcal{E}_{h}$;

(iv) $\int_{F} \boldsymbol{v}_{1} \cdot \boldsymbol{\psi}=\int_{F} \boldsymbol{w} \cdot \boldsymbol{\psi}$

for all $\boldsymbol{\psi} \in \mathcal{P}_{k-6}(F)$ and faces $F \in \mathcal{F}_{h}$;

(v) $\int_{T} \boldsymbol{v}_{1} \cdot \boldsymbol{\phi}=\int_{T} \boldsymbol{I}_{h} \boldsymbol{w} \cdot \boldsymbol{\phi}$, for all $\boldsymbol{\phi} \in \mathcal{P}_{k-4}(T)$ and simplexes $T \in \mathcal{T}_{h}$.

Clearly we have $D^{\alpha}\left(\operatorname{div} \boldsymbol{v}_{1}\right)(a)=D^{\alpha} q(a)$ at all of the vertices and for all multiindices $|\alpha| \leq 1$. Furthermore, by (ii) and Appendix A.

$$
\int_{e} \operatorname{div} \boldsymbol{v}_{1} \sigma=\int_{e}\left(\frac{\partial \boldsymbol{v}_{1}}{\partial \boldsymbol{n}_{e_{+}}} \cdot \boldsymbol{n}_{e_{+}}+\frac{\partial \boldsymbol{v}_{1}}{\partial \boldsymbol{n}_{e_{-}}} \cdot \boldsymbol{n}_{e_{-}}+\frac{\partial \boldsymbol{v}_{1}}{\partial \boldsymbol{t}_{e}} \cdot \boldsymbol{t}_{e}\right) \sigma=\int_{e} q \sigma
$$

for all $\sigma \in \mathcal{P}_{k-5}(e)$. We then find $\operatorname{div} \boldsymbol{v}_{1}=q$ on all edges of the triangulation. Moreover, we have

$$
\int_{T}\left(\operatorname{div} \boldsymbol{v}_{1}\right)=\int_{\partial T} \boldsymbol{v}_{1} \cdot \boldsymbol{n}=\int_{\partial T} \boldsymbol{w} \cdot \boldsymbol{n}=\int_{T} \operatorname{div} \boldsymbol{w}=\int_{T} q .
$$

It then follows that $\left.\left(q-\operatorname{div} \boldsymbol{v}_{1}\right)\right|_{T} \in Q_{k-1,0}(T), \forall T \in \mathcal{T}_{h}$. By Theorem 4.2 and Remark 4.4, there exists $\boldsymbol{v}_{2, T} \in \mathcal{P}_{k}(T) \cap \boldsymbol{H}_{0}^{1}(T)$ such that $\left.\operatorname{div} \boldsymbol{v}_{2, T}\right|_{T}=q-\left.\operatorname{div} \boldsymbol{v}_{1}\right|_{T}$ and $\left\|\boldsymbol{v}_{2, T}\right\|_{H^{1}(T)} \leq C\left(\|q\|_{L^{2}(T)}+\left\|\boldsymbol{v}_{1}\right\|_{H^{1}(T)}\right)$. Define $\boldsymbol{v}_{2} \in \boldsymbol{H}_{0}^{1}(\Omega)$ by $\left.\boldsymbol{v}\right|_{T}=\left.\boldsymbol{v}_{2, T}\right|_{T}$ for all $T \in \mathcal{T}_{h}$. Since $\boldsymbol{v}_{2, T} \in \mathcal{P}_{k}(T) \cap \boldsymbol{H}_{0}^{1}(T)$, we have $D^{\alpha} \boldsymbol{v}_{2, T}(a)=0$ for all vertices of $T$ and for all multi-indices with $|\alpha| \leq 2$. Moreover, $\left.D \boldsymbol{v}_{2, T}\right|_{e}=0$ on all edges of $T$. It then follows that $\boldsymbol{v}_{2} \in \boldsymbol{V}_{h}$. Setting $\boldsymbol{v}=\boldsymbol{v}_{1}+\boldsymbol{v}_{2} \in \boldsymbol{V}_{h}$ we have $\operatorname{div} \boldsymbol{v}=\operatorname{div} \boldsymbol{v}_{1}+$ $\operatorname{div} \boldsymbol{v}_{2}=q$ and $\|\boldsymbol{v}\|_{H^{1}(\Omega)} \leq\left\|\boldsymbol{v}_{1}\right\|_{H^{1}(\Omega)}+\left\|\boldsymbol{v}_{2}\right\|_{H^{1}(\Omega)} \leq C\left(\|q\|_{L^{2}(\Omega)}+\left\|\boldsymbol{v}_{1}\right\|_{H^{1}(\Omega)}\right)$.

To complete the proof we show the estimate $\left\|\boldsymbol{v}_{1}\right\|_{H^{1}(\Omega)} \leq C\|q\|_{L^{2}(\Omega)}$ by a scaling argument. Since $\left.\boldsymbol{I}_{h} \boldsymbol{w}\right|_{T} \in \mathcal{P}_{k}(T)$ for each simplex $T \in \mathcal{T}_{h}$, and since the degrees of freedom (3.2) form a unisolvent set over $\boldsymbol{V}_{h}$, we have by (i)-(v) with $\boldsymbol{z}:=\boldsymbol{v}_{1}-\boldsymbol{I}_{h} \boldsymbol{w}$,

$$
\begin{aligned}
& \|\boldsymbol{z}\|_{H^{1}(T)}^{2} \approx \sum_{a \in \mathcal{V}_{h}(T)}\left\{h_{T}|\boldsymbol{z}(a)|^{2}+h_{T}^{3}|D \boldsymbol{z}(a)|^{2}+h_{T}^{5}\left|D^{2} \boldsymbol{z}(a)\right|^{2}\right\} \\
& +\sum_{e \in \mathcal{E}_{h}(T)}\left\{\left|\sup _{\substack{\boldsymbol{\kappa} \in \mathcal{P}_{k-6}(e) \\
\|\boldsymbol{\kappa}\|_{L^{2}(e)}=1}} \int_{e} \boldsymbol{z} \cdot \boldsymbol{\kappa}\right|^{2}+\left.\left.h_{e}^{2}\right|_{\substack{\boldsymbol{\sigma} \in \mathcal{P}_{k-5}(e) \\
\|\boldsymbol{\sigma}\|_{L^{2}(e)}=1}} \int_{e} \frac{\partial \boldsymbol{z}}{\partial \boldsymbol{n}_{ \pm}} \cdot \boldsymbol{\sigma}\right|^{2}\right\} \\
& +\sum_{F \in \mathcal{F}_{h}(T)} h_{F}^{-1}\left|\sup _{\substack{\boldsymbol{r} \in \mathcal{P}_{k-6}(F) \\
\|\boldsymbol{r}\|_{L^{2}(F)}=1}} \int_{F} \boldsymbol{z} \cdot \boldsymbol{r}\right|^{2}+h_{T}^{-2}\left|\sup _{\substack{\boldsymbol{\varphi} \in \mathcal{P}_{k-4}(T) \\
\|\boldsymbol{\varphi}\|_{L^{2}(T)}=1}} \int_{T} \boldsymbol{z} \cdot \boldsymbol{\varphi}\right|^{2}
\end{aligned}
$$




$$
\begin{aligned}
= & \sum_{a \in \mathcal{V}_{h}(T)}\left\{h_{T}^{3}|D \boldsymbol{z}(a)|^{2}+h_{T}^{5}\left|D^{2} \boldsymbol{z}(a)\right|^{2}\right\}+\sum_{e \in \mathcal{E}_{h}(T)} h_{e}^{2}\left|\sup _{\substack{\boldsymbol{\sigma} \in \mathcal{P}_{k-5}(e) \\
\|\boldsymbol{\sigma}\|_{L^{2}(e)}}} \int_{e} \frac{\partial \boldsymbol{z}}{\partial \boldsymbol{n}_{e_{ \pm}}} \cdot \boldsymbol{\sigma}\right|^{2} \\
& +\sum_{F \in \mathcal{F}_{h}(T)} h_{F}^{-1}\left|\sup _{\substack{\boldsymbol{r} \in \mathcal{P}_{k-6}(F) \\
\|\boldsymbol{r}\|_{L^{2}(e)}=1}} \int_{F}\left(\boldsymbol{w}-\boldsymbol{I}_{h} \boldsymbol{w}\right) \cdot \boldsymbol{r}\right|^{2}
\end{aligned}
$$

By Lemmas 2.3 and 2.1, we have

$$
\begin{aligned}
& \sum_{F \in \mathcal{F}_{h}(T)} h_{F}^{-1}\left|\sup _{\substack{\boldsymbol{r} \in \mathcal{P}_{k-6}(F) \\
\|\boldsymbol{r}\|_{L^{2}(e)}=1}} \int_{F}\left(\boldsymbol{w}-\boldsymbol{I}_{h} \boldsymbol{w}\right) \cdot \boldsymbol{r}\right|^{2} \\
& \quad \leq C h_{T}^{-1}\left\|\boldsymbol{w}-\boldsymbol{I}_{h} \boldsymbol{w}\right\|_{L^{2}(\partial T)}^{2} \leq C\left(h_{T}^{-2}\left\|\boldsymbol{w}-\boldsymbol{I}_{h} \boldsymbol{w}\right\|_{L^{2}(T)}^{2}+\left\|\boldsymbol{w}-\boldsymbol{I}_{h} \boldsymbol{w}\right\|_{H^{1}(T)}^{2}\right) \\
& \quad \leq C\|\boldsymbol{w}\|_{H^{1}(\omega(T))}^{2} .
\end{aligned}
$$

By (i), scaling, and Lemma 2.3, we obtain

$$
\begin{aligned}
& \sum_{a \in \mathcal{V}_{h}(T)}\left\{h_{T}^{3}|D \boldsymbol{z}(a)|^{2}+h_{T}^{5}\left|D^{2} \boldsymbol{z}(a)\right|^{2}\right\} \\
& \quad \leq C \sum_{a \in \mathcal{V}_{h}(T)}\left\{h_{T}^{3}\left|D \boldsymbol{I}_{h} \boldsymbol{w}(a)\right|^{2}+h_{T}^{5}\left|D^{2} \boldsymbol{I}_{h} \boldsymbol{w}(a)\right|^{2}+h_{T}^{3}|q(a)|^{2}+h_{T}^{5}|\nabla q(a)|^{2}\right\} \\
& \quad \leq C\left(\left\|\boldsymbol{I}_{h} \boldsymbol{w}\right\|_{H^{1}(T)}^{2}+\|q\|_{L^{2}(T)}^{2}\right) \leq C\left(\|\boldsymbol{w}\|_{H^{1}(\omega(T))}^{2}+\|q\|_{L^{2}(T)}^{2}\right) .
\end{aligned}
$$

Next, by (i)-(ii) and integration by parts, we deduce that

$$
\int_{e} \frac{\partial \boldsymbol{v}}{\partial \boldsymbol{t}_{e}} \cdot \boldsymbol{t}_{e} \sigma=\int_{e} \frac{\partial \boldsymbol{I}_{h} \boldsymbol{w}}{\partial \boldsymbol{t}_{e}} \cdot \boldsymbol{t}_{e} \sigma
$$

for all $\sigma \in \mathcal{P}_{k-5}(e)$. Therefore by (iii), Lemmas 2.1 and 2.3, and an inverse estimate,

$$
\begin{gathered}
\sum_{e \in \mathcal{E}_{h}(T)} h_{e}^{2}\left|\sup _{\substack{\boldsymbol{\sigma} \in \mathcal{P}_{k-5}(e) \\
\|\boldsymbol{\sigma}\|_{L^{2}(e)}=1}} \int_{e} \frac{\partial \boldsymbol{z}}{\partial \boldsymbol{n}_{e_{ \pm}}} \cdot \boldsymbol{\sigma}\right|^{2} \leq \sum_{e \in \mathcal{E}_{h}(T)} h_{e}^{2}\left(\left\|D \boldsymbol{I}_{h} \boldsymbol{w}\right\|_{L^{2}(e)}^{2}+\|q\|_{L^{2}(e)}^{2}\right) \\
\leq C\left(\left\|\boldsymbol{I}_{h} \boldsymbol{w}\right\|_{H^{1}(T)}^{2}+\|q\|_{L^{2}(T)}^{2}\right) \leq C\left(\|\boldsymbol{w}\|_{H^{1}(\omega(T))}^{2}+\|q\|_{L^{2}(T)}^{2}\right) .
\end{gathered}
$$

Applying the estimates (4.5)-(4.7) to (4.4), we obtain $\left\|\boldsymbol{v}_{1}-\boldsymbol{I}_{h} \boldsymbol{w}\right\|_{H^{1}(T)} \leq$ $C\left(\|\boldsymbol{w}\|_{H^{1}(\omega(T))}+\|q\|_{L^{2}(T)}\right)$. It then follows from the triangle inequality and Lemma 2.3 that $\left\|\boldsymbol{v}_{1}\right\|_{H^{1}(T)} \leq C\left(\|\boldsymbol{w}\|_{H^{1}(\omega(T))}+\|q\|_{L^{2}(T)}\right)$. Summing over $T \in \mathcal{T}_{h}$ and recalling $\|\boldsymbol{w}\|_{H^{1}(\Omega)} \leq C\|q\|_{L^{2}(\Omega)}$, we obtain $\left\|\boldsymbol{v}_{1}\right\|_{H^{1}(\Omega)} \leq C\|q\|_{L^{2}(\Omega)}$, and therefore $\|\boldsymbol{v}\|_{H^{1}(\Omega)} \leq C\|q\|_{L^{2}(\Omega)}$.

Corollary 4.6. The inf-sup (LBB) condition

$$
\sup _{\boldsymbol{v} \in \boldsymbol{V}_{h} \backslash\{0\}} \frac{\int_{\Omega}(\operatorname{div} \boldsymbol{v}) q}{\|\boldsymbol{v}\|_{H^{1}(\Omega)}} \geq C\|q\|_{L^{2}(\Omega)} \quad \forall q \in Q_{h}
$$

holds for a constant $C>0$ independent of $h$.

Corollary 4.7. The image of $\boldsymbol{V}_{h}$ under the divergence operator is $Q_{h}$; that is, $\operatorname{div} \boldsymbol{V}_{h}=Q_{h}$.

Proof. Since $\operatorname{div} \boldsymbol{v}$ is $C^{1}$ at vertices and $C^{0}$ across edges for $\boldsymbol{v} \in \boldsymbol{V}_{h}$, we have $\operatorname{div} \boldsymbol{V}_{h} \subseteq Q_{h}$. On the other hand, Lemma 4.5 gives us the reverse relation $Q_{h} \subseteq$ $\operatorname{div} \boldsymbol{V}_{h}$. The result now follows. 
Theorem 4.8. The complex (1.3) is exact provided $k \geq 7$.

Proof. We show that if $\boldsymbol{v} \in \boldsymbol{V}_{h}$ satisfies $\operatorname{div} \boldsymbol{v}=0$ then $\boldsymbol{v}=\operatorname{curl} \boldsymbol{z}$ for some $\boldsymbol{z} \in \boldsymbol{\Upsilon}_{h}$. Combining this result with Corollary 4.7 proves the theorem.

By (3.4) and Theorem 3.5 we can construct $\boldsymbol{z}_{1} \in \boldsymbol{\Upsilon}_{h}$ such that $\boldsymbol{z}_{1}$ vanishes at the degrees of freedom (3.4b), 3.4d , and $(3.4 \mathrm{~g})$ and

(i) $\boldsymbol{z}_{1}(a)=0$ and $D^{\alpha} \operatorname{curl} \boldsymbol{z}_{1}(a)=D^{\alpha} \boldsymbol{v}(a)$ for all multi-indices $|\alpha| \leq 2$, and vertices $a \in \mathcal{V}_{h}$;

(ii) $D^{\alpha} \operatorname{curl} \boldsymbol{z}_{1}=D^{\alpha} \boldsymbol{v}$ on all edges $e \in \mathcal{E}_{h}$ and multi-indices $|\alpha| \leq 1$;

(iii) $\int_{F}\left(\operatorname{curl} z_{1} \times \boldsymbol{n}_{F}\right) \cdot \boldsymbol{\psi}=\int_{F}\left(\boldsymbol{v} \times \boldsymbol{F}_{F}\right) \cdot \boldsymbol{\psi}$ for all $\boldsymbol{\psi} \in \mathcal{P}_{k-6}(F)$ and faces

Since the value of $\boldsymbol{z}_{1}$ on all edges is uniquely determined by conditions (i)-(iii), we may further use the degrees of freedom (3.4e to impose the condition

(iv) $\int_{F}\left(\boldsymbol{z}_{1} \times \boldsymbol{n}_{F}\right) \cdot \operatorname{curl}_{F} q=\int_{\partial F}\left(\boldsymbol{z}_{1} \cdot \boldsymbol{t}\right) q+\int_{F}\left(\boldsymbol{v} \cdot \boldsymbol{n}_{F}\right) q$ for all $q \in \mathcal{P}_{k-6}(F)$ and

Since $D^{\alpha} \operatorname{curl} \boldsymbol{z}_{1}=D^{\alpha} \boldsymbol{v}$ on $\partial F$, condition (iii) implies curl $\boldsymbol{z}_{1} \times \boldsymbol{n}_{F}=\boldsymbol{v} \times \boldsymbol{n}_{F}$ on all faces $F \in \mathcal{F}_{h}$. By the identity (3.5) and (iv), we have $\int_{F}\left(\operatorname{curl}_{F} \boldsymbol{z}_{1, F}\right) q=$ $\int_{F}\left(\boldsymbol{v} \cdot \boldsymbol{n}_{F}\right) q$ for all $q \in \mathcal{P}_{k-6}(F)$ and $F \in \mathcal{F}_{h}$. Since $\operatorname{curl}_{F} \boldsymbol{z}_{1, F}=\left(\operatorname{curl} \boldsymbol{z}_{1}\right) \cdot \boldsymbol{n}$ and $\left.D^{\alpha}\left(\operatorname{curl} \boldsymbol{z}_{1}-\boldsymbol{v}\right)\right|_{F} \in \mathcal{P}_{k}(F)$ vanishes on $\partial F(|\alpha| \leq 1)$, we have $\left(\operatorname{curl} \boldsymbol{z}_{1}\right) \cdot \boldsymbol{n}=\boldsymbol{v} \cdot \boldsymbol{n}$ on each $F \in \mathcal{F}_{h}$.

Therefore $\left.\left(\boldsymbol{v}-\operatorname{curl} \boldsymbol{z}_{1}\right)\right|_{T} \in \boldsymbol{V}_{k, 0}(T)$ for all $T \in \mathcal{T}_{h}$. By Lemma 4.1 there exists $\boldsymbol{z}_{2, T} \in \boldsymbol{\Upsilon}_{k+1,0}(T)$ such that $\operatorname{curl} \boldsymbol{z}_{2, T}=\left.\left(\boldsymbol{v}-\operatorname{curl} \boldsymbol{z}_{1}\right)\right|_{T}$. Let $\boldsymbol{z}_{2}$ be defined by $\left.\boldsymbol{z}_{2}\right|_{T}=\boldsymbol{z}_{2, T} \forall T \in \mathcal{T}_{h}$. Note that $\boldsymbol{z}_{2, T}$ and $\operatorname{curl} \boldsymbol{z}_{2, T}$ vanish up to first order on the edges of $T$. Write $\boldsymbol{z}_{2, T}=b_{T} \boldsymbol{p}$ for some $\boldsymbol{p} \in \mathcal{P}_{k-5}(T)$. Restricting the identity $\operatorname{curl} \boldsymbol{z}_{2, T}=\operatorname{grad} b_{T} \times \boldsymbol{p}+b_{T} \operatorname{curl} \boldsymbol{p}$ to the boundary $\partial T$ and using the identity (2.1), we have $\boldsymbol{p} \times\left.\boldsymbol{n}\right|_{\partial T}=0$. Therefore $\boldsymbol{p}$ vanishes at the vertices of $T$, and hence $\boldsymbol{z}_{2, T}$ vanishes up to third order at the vertices. It then follows that $\boldsymbol{z}_{2} \in \boldsymbol{\Upsilon}_{h}$. Setting $\boldsymbol{z}:=\boldsymbol{z}_{1}+\boldsymbol{z}_{2}$, we have $\boldsymbol{z} \in \boldsymbol{\Upsilon}_{h}$ and $\operatorname{curl} \boldsymbol{z}=\boldsymbol{v}$.

Remark 4.9. The $H^{2}$-conforming finite element space $\Sigma_{h}$ is only defined for $k \geq 7$, thus leading to the same restriction in Theorem 4.8. However, the finite element spaces $\boldsymbol{\Upsilon}_{h}, \boldsymbol{V}_{h}$ and $Q_{h}$ are well defined with $k=6$, and the exactness property of these spaces are preserved in this case.

\section{Approximation properties of the finite Element spaces}

Since the degrees of freedom (3.2) involve high-order derivatives, the associated canonical projections are not well defined on $\boldsymbol{H}^{1}(\Omega)$. Similarly, the degrees of freedom (3.1), (3.4), and (3.3) are not well defined on $H^{2}(\Omega), \boldsymbol{H}^{1}(\mathbf{c u r l} ; \Omega)$ and $L^{2}$ respectively. Here, we construct an interpolation operator using an averaging technique which is well defined on the appropriate spaces. For brevity we only construct an interpolation operator onto $\boldsymbol{V}_{h}$ bounded in $\boldsymbol{H}^{1}$. Interpolation operators for the other spaces can be constructed by similar arguments.

To define an interpolation operator on the finite element space $\boldsymbol{V}_{h}$, we first derive an auxiliary enriching operator [5]. Let $\omega(a)$ (resp., $\omega(e)$ ) denote the set of 
tetrahedrons which have $a$ (resp., e) as a vertex (resp., edge). We then define the average of a piecewise continuous function $w$ on vertices and edges as

$$
w_{a}=\frac{1}{\left|\mathcal{T}_{a}\right|} \sum_{T \in \omega(a)} w_{T}(a), \quad w_{e}=\left.\frac{1}{\left|\mathcal{T}_{e}\right|} \sum_{T \in \omega(e)} w_{T}\right|_{e}
$$

where $w_{T}:=\left.w\right|_{T}$ and $\left|\mathcal{T}_{a}\right|$ and $\left|\mathcal{T}_{e}\right|$ denote, respectively, the number of tetrahedrons in $\omega(a)$ and $\omega(e)$.

Let $\mathcal{P}_{k}$ denote the globally continuous vector-valued Lagrange finite element space of degree $k$. We then define $\boldsymbol{E}_{h}: \mathcal{P}_{k} \rightarrow \boldsymbol{V}_{h}$ by the following set of conditions:

$$
\begin{array}{rlrl}
D^{\alpha} \boldsymbol{E}_{h} \boldsymbol{w}(a) & =\left(D^{\alpha} \boldsymbol{w}\right)_{a} & & \forall|\alpha| \leq 2, \forall a \in \mathcal{V}_{h}, \\
\int_{e} \boldsymbol{E}_{h} \boldsymbol{w} \cdot \boldsymbol{\kappa}=\int_{e} \boldsymbol{w} \cdot \boldsymbol{\kappa} & \forall \boldsymbol{\kappa} \in \mathcal{P}_{k-6}(e), \forall e \in \mathcal{E}_{h}, \\
\int_{e} \frac{\partial\left(\boldsymbol{E}_{h} \boldsymbol{w}\right)}{\partial \boldsymbol{n}_{e_{ \pm}}} \cdot \boldsymbol{\sigma}=\int_{e}\left(\frac{\partial \boldsymbol{w}}{\partial \boldsymbol{n}_{e_{ \pm}}}\right)_{e} \cdot \boldsymbol{\sigma} & \forall \boldsymbol{\sigma} \in \mathcal{P}_{k-5}(e), \forall e \in \mathcal{E}_{h}, \\
\int_{F} \boldsymbol{E}_{h} \boldsymbol{w} \cdot \boldsymbol{\psi}=\int_{F} \boldsymbol{w} \cdot \boldsymbol{\psi} & \forall \boldsymbol{\psi} \in \mathcal{P}_{k-6}(F), \forall F \in \mathcal{F}_{h}, \\
\int_{T} \boldsymbol{E}_{h} \boldsymbol{w} \cdot \boldsymbol{\varphi}=\int_{T} \boldsymbol{w} \cdot \boldsymbol{\varphi} & \forall \boldsymbol{\varphi} \in \mathcal{P}_{k-4}(T), \forall T \in \mathcal{T}_{h} .
\end{array}
$$

By scaling and since $\boldsymbol{w}$ is globally continuous, we have

$$
\begin{aligned}
\left\|\boldsymbol{E}_{h} \boldsymbol{w}-\boldsymbol{w}\right\|_{L^{2}(T)}^{2} \approx & \sum_{a \in \mathcal{V}_{h}(T)}\left\{h_{T}^{5}\left|\nabla \boldsymbol{E}_{h} \boldsymbol{w}(a)-\nabla \boldsymbol{w}_{T}(a)\right|^{2}+h_{T}^{7}\left|D^{2} \boldsymbol{E}_{h} \boldsymbol{w}(a)-D^{2} \boldsymbol{w}_{T}(a)\right|^{2}\right\} \\
& +\sum_{e \in \mathcal{E}_{h}(T)} h_{e}^{4}\left|\sup _{\substack{\boldsymbol{\sigma} \in \mathcal{P}_{k-5}(e) \\
\|\boldsymbol{\sigma}\|_{L^{2}(e)}=1}} \int_{e} \frac{\partial\left(\boldsymbol{E}_{h} \boldsymbol{w}-\boldsymbol{w}_{T}\right)}{\partial \boldsymbol{n}_{e_{ \pm}}} \cdot \boldsymbol{\sigma}\right|^{2} .
\end{aligned}
$$

Given $T, T^{\prime} \in \omega(a)$, there exists a subset $\left\{T_{i}\right\}_{i=0}^{N} \subset \omega(a)$ with $T_{0}=T, T_{N}=T^{\prime}$, and $T_{i}$ and $T_{i+1}$ share a common face $F_{i}:=\partial T_{i} \cap \partial T_{i+1}$. It then follows from the triangle inequality and standard inverse estimates that

$$
\begin{aligned}
& \left|\nabla \boldsymbol{w}_{T^{\prime}}(a)-\nabla \boldsymbol{w}_{T}(a)\right|^{2} \\
& \quad \leq C \sum_{i=0}^{N-1}\left|\nabla \boldsymbol{w}_{T_{i+1}}(a)-\nabla \boldsymbol{w}_{T_{i}}(a)\right|^{2} \leq C \sum_{i=0}^{N-1}\left\|\nabla \boldsymbol{w}_{T_{i+1}}-\nabla \boldsymbol{w}_{T_{i}}\right\|_{L^{\infty}\left(F_{i}\right)}^{2} \\
& \quad \leq C h_{T}^{-2} \sum_{i=0}^{N-1}\left\|\nabla \boldsymbol{w}_{T_{i+1}}-\nabla \boldsymbol{w}_{T_{i}}\right\|_{L^{2}\left(F_{i}\right)}^{2}=C h_{T}^{-2} \sum_{i=0}^{N-1}\|\llbracket \nabla \boldsymbol{w} \rrbracket\|_{L^{2}\left(F_{i}\right)}^{2},
\end{aligned}
$$

where the jump of $\nabla \boldsymbol{w}$ across $F_{i}$ is defined as $\llbracket \nabla \boldsymbol{w} \rrbracket\left|F_{F_{i}}=\nabla \boldsymbol{w}_{T_{i+1}}\right|_{F_{i}}-\left.\nabla \boldsymbol{w}_{T_{i}}\right|_{F_{i}}$. Combining the estimates (5.1a) and (5.3) we obtain

$$
\begin{aligned}
\left|\nabla \boldsymbol{E}_{h} \boldsymbol{w}(a)-\nabla \boldsymbol{w}_{T}(a)\right|^{2} & =\frac{1}{\left|\mathcal{T}_{a}\right|} \sum_{T^{\prime} \in \omega(a)}\left|\nabla \boldsymbol{w}_{T^{\prime}}(a)-\nabla \boldsymbol{w}_{T}(a)\right|^{2} \\
& \leq C h_{T}^{-2} \sum_{\substack{T^{\prime}, T^{\prime \prime} \in \omega(a) \\
T^{\prime} \cap T^{\prime \prime}=F \neq \emptyset}}\|\llbracket \nabla \boldsymbol{w} \rrbracket\|_{L^{2}(F)}^{2} .
\end{aligned}
$$


By similar arguments, we also have

$$
\left|D^{2} \boldsymbol{E}_{h} \boldsymbol{w}(a)-D^{2} \boldsymbol{w}_{T}(a)\right|^{2} \leq C h_{T}^{-2} \sum_{\substack{T^{\prime}, T^{\prime \prime} \in \omega(a) \\ T^{\prime} \cap T^{\prime \prime}=F \neq \emptyset}}\left\|\left[\left[D^{2} \boldsymbol{w}\right]\right]\right\|_{L^{2}(F)}^{2} .
$$

Next, for $T, T^{\prime} \in \omega(e)$, there exists $\left\{T_{j}\right\}_{j=0}^{N} \subset \omega(e)$ with $T_{0}=T, T_{M}=T^{\prime}$, and $T_{j}$ and $T_{j+1}$ share a common face $F_{j}:=\partial T_{j} \cap \partial T_{j+1}$. Using similar arguments as those found above, we find

$$
\left.\frac{\partial\left(\boldsymbol{w}_{T^{\prime}}-\boldsymbol{w}_{T}\right)}{\partial \boldsymbol{n}_{e_{ \pm}}}\right|_{e} \leq C \sum_{j=0}^{M-1}\left\|\nabla\left(\boldsymbol{w}_{T_{j+1}}-\boldsymbol{w}_{T_{j}}\right)\right\|_{L^{\infty}\left(F_{j}\right)} \leq C h_{T}^{-1} \sum_{j=0}^{M-1}\|\llbracket \nabla \boldsymbol{w} \rrbracket\|_{L^{2}\left(F_{i}\right)} .
$$

By (5.1b) and (5.6), we obtain

$$
\begin{aligned}
& \left|\sup _{\substack{\boldsymbol{\sigma} \in \mathcal{P}_{k-5}(e) \\
\|\boldsymbol{\sigma}\|_{L^{2}(e)}=1}} \int_{e} \frac{\partial\left(\boldsymbol{E}_{h} \boldsymbol{w}-\boldsymbol{w}_{T}\right)}{\partial \boldsymbol{n}_{e_{ \pm}}} \cdot \boldsymbol{\sigma}\right|^{2} \\
& \quad \leq\left\|\partial\left(\boldsymbol{E}_{h} \boldsymbol{w}-\boldsymbol{w}_{T}\right) / \partial \boldsymbol{n}_{e_{ \pm}}\right\|_{L^{2}(e)}^{2} \leq h_{e}\left\|\partial\left(\boldsymbol{E}_{h} \boldsymbol{w}-\boldsymbol{w}_{T}\right) / \partial \boldsymbol{n}_{e_{ \pm}}\right\|_{L^{\infty}(e)}^{2} \\
& \quad \leq h_{T}^{-1} \sum_{\substack{T^{\prime}, T^{\prime \prime} \in \omega(e) \\
T^{\prime} \cap T^{\prime \prime}=F \neq \emptyset}}\|\llbracket \nabla \boldsymbol{w} \rrbracket\|_{L^{2}(F)}^{2} .
\end{aligned}
$$

Applying the estimates (5.4), (5.5), and (5.7) to (5.2) and noting $\bigcup_{e \in \mathcal{E}_{h}(T)} \omega(e) \subset$ $\bigcup_{a \in \mathcal{V}_{h}(T)} \omega(a)$, we obtain

$$
\begin{aligned}
& \left\|\boldsymbol{E}_{h} \boldsymbol{w}-\boldsymbol{w}\right\|_{L^{2}(T)}^{2} \\
& \quad \leq C \sum_{a \in \mathcal{V}_{h}(T)} \sum_{\substack{T^{\prime}, T^{\prime \prime} \in \omega(a) \\
T^{\prime} \cap T^{\prime \prime}=F \neq \emptyset}}\left(h_{T}^{3}\|\llbracket \nabla \boldsymbol{w} \rrbracket\|_{L^{2}(F)}^{2}+h_{T}^{5}\left\|\left[\left[D^{2} \boldsymbol{w}\right]\right]\right\|_{L^{2}(F)}^{2}\right) .
\end{aligned}
$$

Next, given $\boldsymbol{v} \in \boldsymbol{H}^{m}(\Omega)(1 \leq m \leq k+1)$, let $\boldsymbol{I}_{h} \boldsymbol{v}$ be the Scott-Zhang interpolant defined in Lemma 2.3. By (2.5), (2.2) and scaling, we have

$$
\sum_{F \subset \partial T}\left(h_{T}^{3}\left\|\llbracket \nabla\left(\boldsymbol{I}_{h} \boldsymbol{v}\right) \rrbracket\right\|_{L^{2}(F)}^{2}+h_{T}^{5}\left\|\left[\left[D^{2}\left(\boldsymbol{I}_{h} \boldsymbol{v}\right)\right]\right]\right\|_{L^{2}(F)}^{2}\right) \leq C h_{T}^{2 m}\|\boldsymbol{v}\|_{H^{m}(\omega(T))}^{2} .
$$

Finally, we define $\boldsymbol{\Pi}_{h}: \boldsymbol{H}^{1}(\Omega) \rightarrow \boldsymbol{V}_{h}$ by $\boldsymbol{\Pi}_{h}=\boldsymbol{E}_{h} \boldsymbol{I}_{h}$. Then by the triangle inequality we have $(s=0,1)$,

$$
\begin{aligned}
\| \boldsymbol{v}- & \boldsymbol{\Pi}_{h} \boldsymbol{v}\left\|_{H^{s}(T)}=\right\| \boldsymbol{v}-\boldsymbol{E}_{h} \boldsymbol{I}_{h} \boldsymbol{v} \|_{H^{s}(T)} \\
& \leq\left\|\boldsymbol{v}-\boldsymbol{I}_{h} \boldsymbol{v}\right\|_{H^{s}(T)}+C h_{T}^{-s}\left\|\boldsymbol{I}_{h} \boldsymbol{v}-\boldsymbol{E}_{h} \boldsymbol{I}_{h} \boldsymbol{v}\right\|_{L^{2}(T)} \\
& \leq\left\|\boldsymbol{v}-\boldsymbol{I}_{h} \boldsymbol{v}\right\|_{H^{s}(T)}+C h_{T}^{-s}\left(\sum _ { \substack { a \in \mathcal { V } _ { h } ( T ) \\
\begin{subarray} { c } { T ^ { \prime } , T ^ { \prime \prime } \in \omega ( a ) \\
T ^ { \prime } \cap T ^ { \prime \prime } = F \neq \emptyset } } \end{subarray} } \left(h_{T}^{3}\left\|\llbracket \nabla\left(\boldsymbol{I}_{h} \boldsymbol{v}\right) \rrbracket\right\|_{L^{2}(F)}^{2}\right.\right. \\
& \left.+h_{T}^{5} \|\left[\left[D^{2}\left(\boldsymbol{I}_{h} \boldsymbol{v}\right)\right] \|_{L^{2}(F)}^{2}\right)\right)^{1 / 2} .
\end{aligned}
$$


Combining this identity with (5.8) and Lemma 2.3, we obtain the main result of this section.

Lemma 5.1. There exists an operator $\boldsymbol{\Pi}_{h}: \boldsymbol{H}^{1}(\Omega) \rightarrow \boldsymbol{V}_{h}$ such that for any $\boldsymbol{v} \in$ $\boldsymbol{H}^{m}(\Omega)$ with $1 \leq m \leq k+1$, there holds $(0 \leq s \leq 1)$

$$
\left\|\boldsymbol{v}-\boldsymbol{\Pi}_{h} \boldsymbol{v}\right\|_{H^{s}(T)} \leq C h_{T}^{m-s}\|\boldsymbol{v}\|_{H^{m}(\omega(T))} .
$$

\section{IMPOSING HOMOGENEOUS BOUNDARY CONDITIONS AND THE RELATION WITH The SCOtT-Vogelius ELEMENT}

Constructing the analogous exact finite element spaces with homogeneous boundary conditions is a non-trivial issue. For example, we cannot simply take $\boldsymbol{V}_{h, 0}:=$ $\boldsymbol{V}_{h} \cap \boldsymbol{H}_{0}^{1}(\Omega)$ and $Q_{h, 0}:=Q_{h} \cap \stackrel{\circ}{L}^{2}(\Omega)$ to be the last two spaces in the discrete version of (1.2). To see this, note that $D^{\alpha} \boldsymbol{v}(a)=0$ for all multi-indices $|\alpha| \leq 2$ and all corner vertices of the domain $\Omega$. Therefore, $\operatorname{div} \boldsymbol{v}=0$ and its gradient vanish at these points. Since functions in $Q_{h, 0}$ do not necessarily vanish at the corners, the divergence operator div : $\boldsymbol{V}_{h, 0} \rightarrow Q_{h, 0}$ cannot be surjective and therefore the inf-sup condition (4.8) is lost. Similar problems occur on the boundary $\partial \Omega$ where two non-coplanar faces intersect.

A similar situation arises in the construction of the two-dimensional Stokes elements proposed in 15. Here, following a smooth de Rham complex, we use Hermite finite elements to construct stable Stokes elements with pointwise mass conservation. To overcome the difficulty at the corners of the domain, we relax the $C^{1}$ continuity condition of the Hermite elements at these points. A mesh condition is also assumed, namely, that no triangle in the mesh has more than one boundary edge (equivalently, no corner vertices in the mesh are singular). The stability argument of the Scott-Vogelius element [23. $\mathcal{P}_{k} / \mathcal{P}_{k-1}^{d c}(k \geq 4)$ is then used locally at these points to show stability of the finite element pair (see [15, Lemma 3.3] for details). Here, $\mathcal{P}_{k-1}^{d c}$ is the space of piecewise polynomials with no continuity constraints and with degree not exceeding $k-1$, and we recall $\mathcal{P}_{k}$ is the space of globally continuous polynomials with degree $\leq k$.

It is likely that a similar argument can used in the three-dimensional setting, but unfortunately the stability analysis of the three-dimensional Scott-Vogelius pair $\mathcal{P}_{k} / \mathcal{P}_{k-1}^{d c}$ has been an open problem for nearly 30 years (for partial results see [30, 33]). Here, we reduce the stability of the finite element pair $\mathcal{P}_{k} / \mathcal{P}_{k-1}^{d c}$ to the following conjecture.

Conjecture 6.1. Under certain geometric restrictions of the triangulation, for every $q \in \mathcal{P}_{k-1}^{d c}$, there exits $\boldsymbol{v} \in \mathcal{P}_{k}$ such that $D^{\alpha} \operatorname{div} \boldsymbol{v}(a)=D^{\alpha} q(a)$ at all vertices $a \in \mathcal{V}_{h}$ and for all multi-indices $|\alpha| \leq 1$. Moreover, $\|\boldsymbol{v}\|_{H^{1}(\Omega)} \leq C\|q\|_{L^{2}(\Omega)}$.

Remark 6.2. A two-dimensional version of Conjecture 6.1 was shown to be true in [23, 27] provided the triangulation does not contain any singular vertices.

To precisely state our result, we require a definition.

Definition 6.3. An edge $e$ is called singular if the faces in the triangulation meeting at the edge fall on exactly two planes.

Remark 6.4. The definition of a singular edge is a natural higher dimensional generalization of the definition of a singular vertex defined in [23]. 
Proposition 6.5. Suppose that Conjecture 6.1 holds and the triangulation does not contain any singular edges. Then given any $q \in \mathcal{P}_{k-1}^{d c}$ with $k \geq 6$, there exists $\boldsymbol{v} \in \mathcal{P}_{k}$ such that $\operatorname{div} \boldsymbol{v}=q$ and $\|\boldsymbol{v}\|_{H^{1}(\Omega)} \leq C\|q\|_{L^{2}(\Omega)}$. Consequently, the inf-sup condition

$$
\sup _{\boldsymbol{v} \in \mathcal{P}_{k} \backslash\{0\}} \frac{\int_{\Omega}(\operatorname{div} \boldsymbol{v}) q}{\|\boldsymbol{v}\|_{H^{1}(\Omega)}} \geq C\|q\|_{L^{2}(\Omega)} \quad \forall q \in \mathcal{P}_{k-1}^{d c}
$$

holds for a constant $C>0$ independent of $h$.

To prove Proposition 6.5 we first require a technical result.

Lemma 6.6. Suppose that the triangulation does not contain any singular edges. Then given $q \in \mathcal{P}_{k-1}^{d c}$, there exists $\boldsymbol{v} \in \mathcal{P}_{k}$ such that $\operatorname{div} \boldsymbol{v}$ equals $q$ at $(k-4)$ distinct points on each edge and $\|\boldsymbol{v}\|_{H^{1}(\Omega)} \leq C\|q\|_{L^{2}(\Omega)}$. Moreover, $D^{\alpha} \operatorname{div} \boldsymbol{v}(x)=0$ at all vertices and for all multi-indices $|\alpha| \leq 1$.

Proof. The proof follows the argument given in [27, Lemma 2.6].

First we observe that the degrees of freedom (3.2) can be used to construct finite element spaces that are continuous but not $C^{1}$ across edges of the triangulation. This is achieved by replacing the derivative DOFs (3.2c) by tangential DOFs in a local fashion.

Let $T^{+}$and $T^{-}$be two tetraheda with a common face $\bar{F}=\partial T^{+} \cap \partial T^{-}$, and let $\bar{e}$ be an edge of $\bar{F}$. The construction of $\boldsymbol{v}$ with the desired properties will be associated with the edge $\bar{e}$. For an edge $e$ of $\partial T^{+} \cap \partial T^{-}$, we denote by $F_{e}^{ \pm}$the two faces such that $e=\partial F_{e}^{+} \cap \partial F_{e}^{-}, F_{e}^{ \pm} \neq \bar{F}, F_{e}^{+} \subset \partial T^{+}$and $F_{e}^{-} \subset \partial T^{-}$. Let $\boldsymbol{t}_{e}$ denote a unit vector parallel to the edge $e$, and let $\boldsymbol{s}_{e}^{ \pm}$denote unit tangent vectors of the faces $F_{e}^{ \pm}$with $\boldsymbol{s}_{e}^{ \pm} \cdot \boldsymbol{t}_{e}=0$. We also set $\boldsymbol{\tau}_{e}$ to be a unit tangent of $\bar{F}$ with $\boldsymbol{\tau}_{e} \cdot \boldsymbol{t}_{e}=0$.

Similarly, for an edge $e$ of $\partial T^{+} \cup \partial T^{-} \backslash \bar{F}$, we denote by $F_{e}^{(1)}$ and $F_{e}^{(2)}$ the two faces such that $e=\partial F_{e}^{(1)} \cap \partial F_{e}^{(2)}$. Note that either $F_{e}^{(1)}, F_{e}^{(2)} \subset T^{+}$or $F_{e}^{(1)}, F_{e}^{(2)} \subset T^{-}$. Let $\boldsymbol{t}_{e}$ denote a unit vector parallel to the edge $e$, and let $\boldsymbol{s}_{e}^{(i)}$ denote the unit tangents of the faces $F_{e}^{(i)}(i=1,2)$ with $\boldsymbol{s}_{e}^{(i)} \cdot \boldsymbol{t}_{e}=0$.

We then construct $\boldsymbol{v}$ to be a piecewise polynomial of degree $k$ that is continuous on $T^{+} \cup T^{-}$such that

(i) $D^{\alpha} \boldsymbol{v}(a)=0$ for all $|\alpha| \leq 2$ and $a \in \mathcal{V}_{h}\left(T^{+}\right) \cup \mathcal{V}_{h}\left(T^{-}\right)$;

(ii) $\int_{e} \boldsymbol{v} \cdot \boldsymbol{\kappa}=0$ for all $\kappa \in \mathcal{P}_{k-6}(e)$ and $e \in \mathcal{E}_{h}\left(T^{+}\right) \cup \mathcal{E}_{h}\left(T^{-}\right)$;

(iii) $\int_{F} \boldsymbol{v} \cdot \boldsymbol{r}=0$ for all $\boldsymbol{r} \in \mathcal{P}_{k-6}(F)$ and $F \in \mathcal{F}_{h}\left(T^{+}\right) \cup \mathcal{F}_{h}\left(T^{-}\right)$;

(iv) $\partial \boldsymbol{v} / \partial \boldsymbol{s}_{e}^{(i)}$ vanishes at $(k-4)$ distinct points on each edge of $\partial T^{+} \cup \partial T^{-} \backslash \bar{F}$ $(i=1,2)$;

(v) $\partial \boldsymbol{v}^{ \pm} / \partial \boldsymbol{s}_{e}^{ \pm}$vanishes at $(k-4)$ distinct points on each edge of $\bar{F}$, where $\boldsymbol{v}^{ \pm}:=\left.\boldsymbol{v}\right|_{T^{ \pm}}$

(vi) $\partial \boldsymbol{v} / \partial \boldsymbol{\tau}_{e}$ vanishes at $(k-4)$ distinct points on each edge $e \subset \bar{F}$ with $e \neq \bar{e}$;

(vii) $\int_{T^{ \pm}} \boldsymbol{v} \cdot \boldsymbol{\varphi}=0$ for all $\boldsymbol{\varphi} \in \mathcal{P}_{k-4}\left(T^{ \pm}\right)$.

It remains to specify $\partial \boldsymbol{v} / \partial \boldsymbol{\tau}_{\bar{e}}$ at $(k-4)$ distinct points on the edge $\bar{e}$ to uniquely define $\boldsymbol{v}$. We note at this point that $\left.\nabla \boldsymbol{v}^{ \pm}\right|_{e}=0$ for all edges $e \neq \bar{e}$ and $\left.\boldsymbol{v}\right|_{\partial\left(T^{+} \cup T^{-}\right)}=$ 0 . 
For three linearly independent vectors $\boldsymbol{\alpha}, \boldsymbol{\beta}$, and $\boldsymbol{\gamma}$, we have the identity (cf. Lemma A.1

$$
\operatorname{div} \boldsymbol{v}=\left((\boldsymbol{\beta} \times \boldsymbol{\gamma}) \cdot \frac{\partial \boldsymbol{v}}{\partial \boldsymbol{\alpha}}+(\boldsymbol{\gamma} \times \boldsymbol{\alpha}) \cdot \frac{\partial \boldsymbol{v}}{\partial \boldsymbol{\beta}}+(\boldsymbol{\alpha} \times \boldsymbol{\beta}) \cdot \frac{\partial \boldsymbol{v}}{\partial \boldsymbol{\gamma}}\right) /(\boldsymbol{\alpha} \cdot(\boldsymbol{\beta} \times \boldsymbol{\gamma}))
$$

Since $\frac{\partial \boldsymbol{v}^{+}}{\partial \boldsymbol{s}_{\bar{e}_{+}}}=0$ and $\frac{\partial \boldsymbol{v}}{\partial \boldsymbol{t}_{\bar{e}}}=0$ on edge $\bar{e}$, we have by (6.1) with $\boldsymbol{\alpha}=\boldsymbol{s}_{\bar{e}_{+}}, \boldsymbol{\beta}=\boldsymbol{t}_{\bar{e}}$, and $\gamma=\tau_{\bar{e}}$,

$$
\left.\operatorname{div} \boldsymbol{v}^{+}\right|_{\bar{e}}=\left(\boldsymbol{s}_{\bar{e}_{+}} \times \boldsymbol{t}_{\bar{e}}\right) \cdot \frac{\partial \boldsymbol{v}}{\partial \boldsymbol{\tau}_{\bar{e}}} /\left(\boldsymbol{s}_{\bar{e}_{+}} \cdot\left(\boldsymbol{t}_{\bar{e}} \times \boldsymbol{\tau}_{\bar{e}}\right)\right) .
$$

Similarly, since $\left.\frac{\partial \boldsymbol{v}^{-}}{\partial \boldsymbol{s}_{\bar{e}_{-}}}\right|_{\bar{e}}=0$, we have

$$
\left.\operatorname{div} \boldsymbol{v}^{-}\right|_{\bar{e}}=\left(\boldsymbol{s}_{\bar{e}_{-}} \times \boldsymbol{t}_{\bar{e}}\right) \cdot \frac{\partial \boldsymbol{v}}{\partial \boldsymbol{\tau}_{\bar{e}}} /\left(\boldsymbol{s}_{\bar{e}_{-}} \cdot\left(\boldsymbol{t}_{\bar{e}} \times \boldsymbol{\tau}_{\bar{e}}\right)\right)
$$

It is easy to see that the vectors $\left(\boldsymbol{s}_{\bar{e}_{+}} \times \boldsymbol{t}_{\bar{e}}\right)$ and $\left(\boldsymbol{s}_{\bar{e}_{-}} \times \boldsymbol{t}_{\bar{e}}\right)$ are linearly independent except in the case $\boldsymbol{s}_{\bar{e}_{+}}=-\boldsymbol{s}_{\bar{e}_{-}}$. Since we can specify $\partial \boldsymbol{v} / \partial \boldsymbol{\tau}_{\bar{e}}$ at $(k-4)$ distinct points on the edge $\bar{e}$, we can prescribe two sets of values div $\boldsymbol{v}$ at $(k-4)$ points on edge $\bar{e}$ : one set on $T^{+}$and one set on $T^{-}$.

We can then apply this result to an internal edge $e$. Let $\left\{T_{i}\right\}_{i=1}^{N}$ denote the set of simplices that have $e$ as an edge and labeled such that $T_{i}$ and $T_{i+1}$ share a common face. Since $e$ does not lie on two pairs of co-planar faces, we can assume that the simplices are labeled such that $T_{1}$ and $T_{N}$ do not have faces that have $e$ as an edge and are parallel to each other. We also let $\left\{a_{i}\right\}_{i=1}^{k-4}$ denote $(k-4)$ distinct points on $e$. Then by the procedure just described, we may prescribe $\left.\operatorname{div} \boldsymbol{v}\right|_{T_{1}}\left(a_{i}\right)=\left.\operatorname{div} \boldsymbol{v}\right|_{T_{2}}\left(a_{i}\right)=c_{i}^{(1)}(1 \leq i \leq k-4)$ for some constants $c_{i}^{(1)}$. Similarly, we can prescribe $\left.\operatorname{div} \boldsymbol{v}\right|_{T_{2}}\left(a_{i}\right)$ and $\operatorname{div} \boldsymbol{v}_{T_{2}}\left(a_{i}\right)$ in the same manner. Continuing this procedure for all simplices, and since the edge $e$ does not lie on two pairs of co-planar faces, we have $\left.\operatorname{div} \boldsymbol{v}\right|_{T_{j}}\left(a_{i}\right)=c_{i}^{(j-1)}+c_{i}^{(j)}$ for $1 \leq j \leq N$ and $1 \leq i \leq k-4$. Thus, we may choose the constants $c_{i}^{(j)}$ such that $\left.\operatorname{div} \boldsymbol{v}\right|_{T_{j}}\left(a_{i}\right)=\left.q\right|_{T_{j}}\left(a_{i}\right)$ and $\operatorname{div} \boldsymbol{v}=0$ on all the other edges. A similar construction can be achieved for edges that touch or lie on the boundary. By summing over all edges and using scaling arguments, we obtain the desired result.

Proof of Proposition 6.5. Let $\boldsymbol{v}_{1} \in \mathcal{P}_{k}$ satisfy $D^{\alpha} \operatorname{div} \boldsymbol{v}_{1}(a)=D^{\alpha} q(a)$ at all vertices of the triangulation. Applying Lemma 6.6 with $q$ replaced by $\left(q-\operatorname{div} \boldsymbol{v}_{1}\right)$, there exists $\boldsymbol{v}_{2} \in \mathcal{P}_{k}$ such that $D^{\alpha} \operatorname{div} \boldsymbol{v}_{2}(a)=0$ at all vertices and $|\alpha| \leq 1$, and $\operatorname{div} \boldsymbol{v}_{2}=$ $\left(q-\operatorname{div} \boldsymbol{v}_{1}\right)$ at $(k-4)$ distinct points on each edge. Defining $\overline{\boldsymbol{v}}:=\boldsymbol{v}_{1}+\boldsymbol{v}_{2}$ we see that $D^{\alpha} \operatorname{div} \overline{\boldsymbol{v}}(a)=D^{\alpha} q(a)$ for all $|\alpha| \leq 1$ and $\operatorname{div} \overline{\boldsymbol{v}}=q$ at $(k-4)$ distinct points of each edge. Therefore $\operatorname{div} \overline{\boldsymbol{v}}=q$ on all edges of the triangulation. Moreover, Lemma 6.6 and Conjecture 6.1 give us $\|\overline{\boldsymbol{v}}\|_{H^{1}(\Omega)} \leq C\|q\|_{L^{2}(\Omega)}$.

Next, let $\boldsymbol{w} \in \boldsymbol{H}^{1}(\Omega)$ satisfy $\operatorname{div} \boldsymbol{w}=q$ and $\|\boldsymbol{w}\|_{H^{1}(\Omega)} \leq C\|q\|_{L^{2}(\Omega)}$. Since $\boldsymbol{V}_{h} \subset \mathcal{P}_{k}$, we can construct $\boldsymbol{v}_{3} \in \mathcal{P}_{k}$ such that $\boldsymbol{v}_{3}$ vanishes at the degrees of freedom (3.2a) - (3.2C), (3.2e) and $\int_{F} \boldsymbol{v}_{3} \cdot \boldsymbol{r}=\int_{F}(\boldsymbol{w}-\overline{\boldsymbol{v}}) \cdot \boldsymbol{r}$ for all $\boldsymbol{r} \in \mathcal{P}_{k-6}(F)$ and $F \in \mathcal{F}_{h}$. Then $\boldsymbol{v}_{3}$ and its gradient vanish on all of the edges in the triangulation. Furthermore, Green's formula gives us $\int_{T} \operatorname{div}\left(\overline{\boldsymbol{v}}+\boldsymbol{v}_{3}\right)=\int_{T} q$ for all $T \in \mathcal{T}_{h}$. It then follows that $\left.\left(q-\operatorname{div}\left(\overline{\boldsymbol{v}}+\boldsymbol{v}_{3}\right)\right)\right|_{T} \in Q_{0}(T)(\mathrm{cf}$. (4.1)), and therefore by Theorem 4.2 and Remark 4.4 there exists $\boldsymbol{v}_{4, T} \in \mathcal{P}_{k}(T)$ such that $\left.\operatorname{div} \boldsymbol{v}_{4, T}\right|_{T}=\left.\left(q-\operatorname{div}\left(\overline{\boldsymbol{v}}+\boldsymbol{v}_{3}\right)\right)\right|_{T}$. 
Defining $\boldsymbol{v}=\boldsymbol{v}_{1}+\boldsymbol{v}_{2}+\boldsymbol{v}_{3}+\boldsymbol{v}_{4}$ with $\left.\boldsymbol{v}_{4}\right|_{T}=\left.\boldsymbol{v}_{4, T}\right|_{T}$, we have $\operatorname{div} \boldsymbol{v}=q$. Moreover, by scaling we have $\|\boldsymbol{v}\|_{H^{1}(\Omega)} \leq C\|q\|_{L^{2}(\Omega)}$.

\section{Conclusions}

In this paper, we have constructed conforming finite element spaces in three dimensions with high regularity. By making use of the degrees of freedom and by using an exact local de Rham complex, we have showed that the global discrete complex (1.3) is exact and desirable stability estimates are satisfied.

We end this paper by discussing possible extensions. First, a natural idea is to construct the sequence (1.3) starting with a different $H^{2}$-conforming finite element space. For example, one could take $\Sigma_{h}$ to be the quintic composite element documented in [18]. This element is most likely related to the $\mathcal{P}_{3} / \mathcal{P}_{2}^{d c}$ Stokes pair on barycenter refined triangulations studied in 30. However, the corresponding $\boldsymbol{H}^{1}(\mathbf{c u r l} ; \Omega)$ conforming element in the sequence (1.3) appears to be missing in the literature.

Alternatively, we may look for other smooth de Rham complexes which may lead to simpler finite element spaces. Following the ideas in [15, one may consider the complex with additional regularity

$$
\mathbb{R} \longrightarrow H^{2}(\Omega) \stackrel{\text { grad }}{\longrightarrow} \boldsymbol{H}^{1}(\operatorname{curl} ; \Omega) \stackrel{\text { curl }}{\longrightarrow} \boldsymbol{H}^{1}(\operatorname{div} ; \Omega) \stackrel{\text { div }}{\longrightarrow} H^{1}(\Omega) \longrightarrow 0,
$$

where $\boldsymbol{H}^{1}(\operatorname{div} ; \Omega)$ consists of functions $\boldsymbol{v} \in \boldsymbol{H}^{1}(\Omega)$ such that $\operatorname{div} \boldsymbol{v} \in H^{1}(\Omega)$. Surprisingly, the two-dimensional $\boldsymbol{H}^{1}(\operatorname{div} ; \Omega)$ Stokes elements in [15] have less degrees of freedom than the corresponding $\boldsymbol{H}^{1}$ elements. We expect a similar result in the three-dimensional setting. We may also consider the de Rham complex considered in 13,14

$$
\mathbb{R} \longrightarrow H^{1}(\Omega) \stackrel{\text { grad }}{\longrightarrow} \boldsymbol{\Phi}(\Omega) \stackrel{\text { curl }}{\longrightarrow} \boldsymbol{H}^{1}(\Omega) \stackrel{\text { div }}{\longrightarrow} L^{2}(\Omega) \longrightarrow 0,
$$

where $\boldsymbol{\Phi}(\Omega):=\left\{\boldsymbol{v} \in \boldsymbol{L}^{2}(\Omega), \operatorname{curl} \boldsymbol{v} \in \boldsymbol{H}^{1}(\Omega)\right\}$. Since this sequence involves larger spaces, the corresponding finite elements may have smaller degree and less complexity.

\section{Appendix A. Some Calculus identities}

Lemma A.1. For any any linearly independent vectors $\boldsymbol{\alpha}, \boldsymbol{\beta}, \boldsymbol{\gamma} \in \mathbb{R}^{3}$, there holds

$$
\operatorname{grad} v=\left((\boldsymbol{\beta} \times \boldsymbol{\gamma}) \frac{\partial v}{\partial \boldsymbol{\alpha}}+(\boldsymbol{\gamma} \times \boldsymbol{\alpha}) \frac{\partial v}{\partial \boldsymbol{\beta}}+(\boldsymbol{\alpha} \times \boldsymbol{\beta}) \frac{\partial v}{\partial \boldsymbol{\gamma}}\right) /(\boldsymbol{\alpha} \cdot(\boldsymbol{\beta} \times \boldsymbol{\gamma}))
$$

Consequently,

$$
\operatorname{div} \boldsymbol{v}=\left((\boldsymbol{\beta} \times \boldsymbol{\gamma}) \cdot \frac{\partial \boldsymbol{v}}{\partial \boldsymbol{\alpha}}+(\boldsymbol{\gamma} \times \boldsymbol{\alpha}) \cdot \frac{\partial \boldsymbol{v}}{\partial \boldsymbol{\beta}}+(\boldsymbol{\alpha} \times \boldsymbol{\beta}) \cdot \frac{\partial \boldsymbol{v}}{\partial \boldsymbol{\gamma}}\right) /(\boldsymbol{\alpha} \cdot(\boldsymbol{\beta} \times \boldsymbol{\gamma}))
$$

Proof. Write $\operatorname{grad} v=\boldsymbol{a} \frac{\partial v}{\partial \boldsymbol{\alpha}}+\boldsymbol{b} \frac{\partial v}{\partial \boldsymbol{\beta}}+\boldsymbol{c} \frac{\partial v}{\partial \boldsymbol{\gamma}}$ for some unknown constants $\boldsymbol{a}, \boldsymbol{b}$ and c. We may then write

$$
\operatorname{grad} v=\left(\boldsymbol{a} \boldsymbol{\alpha}^{t}+\boldsymbol{b} \boldsymbol{\beta}^{t}+\boldsymbol{c} \boldsymbol{\gamma}^{t}\right) \operatorname{grad} v \quad \Longrightarrow \quad \boldsymbol{a} \boldsymbol{\alpha}^{t}+\boldsymbol{b} \boldsymbol{\beta}^{t}+\boldsymbol{c} \boldsymbol{\gamma}^{t}=I_{3 \times 3} .
$$

Multiplying the last expression by $(\boldsymbol{\alpha} \times \boldsymbol{\beta})$, we obtain $\boldsymbol{c}(\boldsymbol{\gamma} \cdot(\boldsymbol{\alpha} \times \boldsymbol{\beta}))=\boldsymbol{\alpha} \times \boldsymbol{\beta}$. Therefore $\boldsymbol{c}=\boldsymbol{\alpha} \times \boldsymbol{\beta} /(\boldsymbol{\gamma} \cdot(\boldsymbol{\alpha} \times \boldsymbol{\beta}))$. Similarly, $\boldsymbol{b}=\boldsymbol{\alpha} \times \boldsymbol{\gamma} /(\boldsymbol{\beta} \cdot(\boldsymbol{\alpha} \times \boldsymbol{\gamma}))$, and 
$\boldsymbol{a}=\boldsymbol{\beta} \times \boldsymbol{\gamma} /(\boldsymbol{\alpha} \cdot(\boldsymbol{\beta} \times \boldsymbol{\gamma}))$. The identity A.1) then follows from the identity $\boldsymbol{A} \cdot(\boldsymbol{B} \times$ $\boldsymbol{C})=\boldsymbol{B} \cdot(\boldsymbol{C} \times \boldsymbol{A})=\boldsymbol{C} \cdot(\boldsymbol{A} \times \boldsymbol{B})$.

Lemma A.2. For any orthonormal vectors $\boldsymbol{\alpha}, \boldsymbol{\beta}, \boldsymbol{\gamma} \in \mathbb{R}^{3}$, there holds

$$
\boldsymbol{\alpha} \cdot \frac{\partial(\operatorname{curl} \boldsymbol{v})}{\partial \boldsymbol{\alpha}}+\boldsymbol{\beta} \cdot \frac{\partial(\operatorname{curl} \boldsymbol{v})}{\partial \boldsymbol{\beta}}+\boldsymbol{\gamma} \cdot \frac{\partial(\operatorname{curl} \boldsymbol{v})}{\partial \boldsymbol{\gamma}}=0 .
$$

Proof. Since the vectors are orthonormal, we have $\boldsymbol{\beta} \times \boldsymbol{\gamma}= \pm \boldsymbol{\alpha}$. Therefore by the vector triple product formula, we find $\boldsymbol{\gamma} \times \boldsymbol{\alpha}= \pm \boldsymbol{\beta}$ and $\boldsymbol{\alpha} \times \boldsymbol{\beta}= \pm \boldsymbol{\gamma}$. By Lemma A.1, with $\boldsymbol{v}$ replaced by $\operatorname{curl} \boldsymbol{v}$, we have

$$
\begin{aligned}
\boldsymbol{\alpha} \cdot \frac{\partial(\operatorname{curl} \boldsymbol{v})}{\partial \boldsymbol{\alpha}}+\boldsymbol{\beta} \cdot \frac{\partial(\operatorname{curl} \boldsymbol{v})}{\partial \boldsymbol{\beta}}+\boldsymbol{\gamma} \cdot \frac{\partial(\operatorname{curl} \boldsymbol{v})}{\partial \boldsymbol{\gamma}} \\
=\left((\boldsymbol{\beta} \times \boldsymbol{\gamma}) \cdot \frac{\partial(\operatorname{curl} \boldsymbol{v})}{\partial \boldsymbol{\alpha}}+(\boldsymbol{\gamma} \times \boldsymbol{\alpha}) \cdot \frac{\partial(\operatorname{curl} \boldsymbol{v})}{\partial \boldsymbol{\beta}}\right. \\
\left.\quad+(\boldsymbol{\alpha} \times \boldsymbol{\beta}) \cdot \frac{\partial(\operatorname{curl} \boldsymbol{v})}{\partial \boldsymbol{\gamma}}\right) /(\boldsymbol{\alpha} \cdot(\boldsymbol{\beta} \times \gamma)) \\
=\operatorname{div} \operatorname{curl} \boldsymbol{v}=0
\end{aligned}
$$

\section{REFERENCES}

[1] D. N. Arnold, R. S. Falk, and R. Winther, Finite element exterior calculus, homological techniques, and applications, Acta Numer. 15 (2006), 1-155, DOI 10.1017/S0962492906210018. MR2269741 (2007j:58002)

[2] D. N. Arnold, R. S. Falk, and R. Winther, Finite element exterior calculus: from Hodge theory to numerical stability, Bull. Amer. Math. Soc. (N.S.) 47 (2010), no. 2, 281-354, DOI 10.1090/S0273-0979-10-01278-4. MR2594630(2011f:58005)

[3] D. Boffi, F. Brezzi, L. F. Demkowicz, R. G. Durán, R. S. Falk, and M. Fortin, Mixed Finite Elements, Compatibility Conditions, and Applications, Lecture Notes in Mathematics, vol. 1939, Springer-Verlag, Berlin; Fondazione C.I.M.E., Florence, 2008. Lectures given at the C.I.M.E. Summer School held in Cetraro, June 26-July 1, 2006; Edited by Boffi and Lucia Gastaldi. MR2459075(2010h:65219)

[4] S. C. Brenner and L. R. Scott, The Mathematical Theory of Finite Element Methods, 3rd ed., Texts in Applied Mathematics, vol. 15, Springer, New York, 2008. MR2373954 (2008m:65001)

[5] S. C. Brenner and L.-Y. Sung, $C^{0}$ interior penalty methods for fourth order elliptic boundary value problems on polygonal domains, J. Sci. Comput. 22/23 (2005), 83-118, DOI 10.1007/s10915-004-4135-7. MR2142191 (2005m:65258)

[6] F. Brezzi and M. Fortin, Mixed and Hybrid Finite Element Methods, Springer Series in Computational Mathematics, vol. 15, Springer-Verlag, New York, 1991. MR1115205(92d:65187)

[7] A. Buffa, J. Rivas, G. Sangalli, and R. Vázquez, Isogeometric discrete differential forms in three dimensions, SIAM J. Numer. Anal. 49 (2011), no. 2, 818-844, DOI 10.1137/100786708. MR2792397(2012j:65388)

[8] P. G. Ciarlet, The Finite Element Method for Elliptic Problems, North-Holland Publishing Co., Amsterdam-New York-Oxford, 1978. Studies in Mathematics and its Applications, Vol. 4. MR0520174 (58 \#25001)

[9] J. Carrero, B. Cockburn, and D. Schötzau, Hybridized globally divergence-free LDG methods. I. The Stokes problem, Math. Comp. 75 (2006), no. 254, 533-563 (electronic), DOI 10.1090/S0025-5718-05-01804-1. MR2196980 (2006m:76040)

[10] B. Cockburn, G. Kanschat, and D. Schötzau, A note on discontinuous Galerkin divergencefree solutions of the Navier-Stokes equations, J. Sci. Comput. 31 (2007), no. 1-2, 61-73, DOI 10.1007/s10915-006-9107-7. MR2304270 (2008f:76109)

[11] L. Demkowicz, P. Monk, L. Vardapetyan, and W. Rachowicz, de Rham diagram for hp finite element spaces, Comput. Math. Appl. 39 (2000), no. 7-8, 29-38, DOI 10.1016/S08981221(00)00062-6. MR:1746160 (2000m:78052) 
[12] L. Demkowicz and A. Buffa, $H^{1}, H$ (curl) and $H$ (div)-conforming projection-based interpolation in three dimensions. Quasi-optimal p-interpolation estimates, Comput. Methods Appl. Mech. Engrg. 194 (2005), no. 2-5, 267-296, DOI 10.1016/j.cma.2004.07.007. MR2105164 (2005j:65139)

[13] J. A. Evans and T. J. R. Hughes, Isogeometric divergence-conforming B-splines for the steady Navier-Stokes equations, Math. Models Methods Appl. Sci. 23 (2013), no. 8, 1421-1478, DOI 10.1142/S0218202513500139. MR3048532

[14] J. A. Evans and T. J. R. Hughes, Isogeometric divergence-conforming B-splines for the steady Navier-Stokes equations, Math. Models Methods Appl. Sci. 23 (2013), no. 8, 1421-1478, DOI 10.1142/S0218202513500139. MR.3048532

[15] R. S. Falk and M. Neilan, Stokes complexes and the construction of stable finite elements with pointwise mass conservation, SIAM J. Numer. Anal. 51 (2013), no. 2, 1308-1326, DOI 10.1137/120888132. MR 3045658

[16] V. Girault and P.-A. Raviart, Finite Element Methods for Navier-Stokes Equations: Theory and algorithms, Springer Series in Computational Mathematics, vol. 5, Springer-Verlag, Berlin, 1986. MR851383 (88b:65129)

[17] J. Guzmán and M. Neilan, A family of nonconforming elements for the Brinkman problem, IMA J. Numer. Anal. 32 (2012), no. 4, 1484-1508, DOI 10.1093/imanum/drr040. MR2991835

[18] M.-J. Lai and L. L. Schumaker, Spline Functions on Triangulations, Encyclopedia of Mathematics and its Applications, vol. 110, Cambridge University Press, Cambridge, 2007. MR2355272(2008i:41001)

[19] J. Li, T. Arbogast, and Y. Huang, Mixed methods using standard conforming finite elements, Comput. Methods Appl. Mech. Engrg. 198 (2009), no. 5-8, 680-692, DOI 10.1016/j.cma.2008.10.002. MR.2498523 (2010a:65241)

[20] K. A. Mardal, X.-C. Tai, and R. Winther, A robust finite element method for Darcy-Stokes flow, SIAM J. Numer. Anal. 40 (2002), no. 5, 1605-1631, DOI 10.1137/S0036142901383910. MR.1950614 (2003m:76110)

[21] P. Monk, Finite Element Methods for Maxwell's Equations, Numerical Mathematics and Scientific Computation, Oxford University Press, New York, 2003. MR 2059447(2005d:65003)

[22] J.-C. Nédélec, Mixed finite elements in $\mathbf{R}^{3}$, Numer. Math. 35 (1980), no. 3, 315-341, DOI 10.1007/BF01396415. MR592160 (81k:65125)

[23] L. R. Scott and M. Vogelius, Norm estimates for a maximal right inverse of the divergence operator in spaces of piecewise polynomials (English, with French summary), RAIRO Modél. Math. Anal. Numér. 19 (1985), no. 1, 111-143. MR813691 (87i:65190)

[24] L. R. Scott and S. Zhang, Finite element interpolation of nonsmooth functions satisfying boundary conditions, Math. Comp. 54 (1990), no. 190, 483-493, DOI 10.2307/2008497. MR.1011446 (90j:65021)

[25] J. Sun, A mixed finite element method for the quad-curl eigenvalue problem, arXiv:1310.6618v1.

[26] X.-C. Tai and R. Winther, A discrete de Rham complex with enhanced smoothness, Calcolo 43 (2006), no. 4, 287-306, DOI 10.1007/s10092-006-0124-6. MR2283095 (2007j:76050)

[27] M. Vogelius, A right-inverse for the divergence operator in spaces of piecewise polynomials. Application to the p-version of the finite element method, Numer. Math. 41 (1983), no. 1, 19-37, DOI 10.1007/BF01396303. MR696548 (85f:65113a)

[28] X. Xu and S. Zhang, A new divergence-free interpolation operator with applications to the Darcy-Stokes-Brinkman equations, SIAM J. Sci. Comput. 32 (2010), no. 2, 855-874, DOI 10.1137/090751049. MR2609343 (2011g:76108)

[29] A. Ženíšek, Polynomial approximation on tetrahedrons in the finite element method, J. Approximation Theory 7 (1973), 334-351. MR0350260 (50 \#2753)

[30] S. Zhang, A new family of stable mixed finite elements for the $3 D$ Stokes equations, Math. Comp. 74 (2005), no. 250, 543-554, DOI 10.1090/S0025-5718-04-01711-9. MR2114637 (2005j:65151)

[31] S. Zhang, On the P1 Powell-Sabin divergence-free finite element for the Stokes equations, J. Comput. Math. 26 (2008), no. 3, 456-470. MR2421893 (2009j:76160)

[32] S. Zhang, A family of $3 D$ continuously differentiable finite elements on tetrahedral grids, Appl. Numer. Math. 59 (2009), no. 1, 219-233, DOI 10.1016/j.apnum.2008.02.002. MR2474112(2010a:65250) 
[33] S. Zhang, Divergence-free finite elements on tetrahedral grids for $k \geq 6$, Math. Comp. 80 (2011), no. 274, 669-695, DOI 10.1090/S0025-5718-2010-02412-3. MR2772092 (2012b:65191)

[34] B. Zheng, Q. Hu, and J. Xu, A nonconforming finite element method for fourth order curl equations in $\mathbb{R}^{3}$, Math. Comp. 80 (2011), no. 276, 1871-1886, DOI 10.1090/S0025-5718-201102480-4. MR2813342 (2012g:65216)

Department of Mathematics, University of Pittsburgh, Pittsburgh, Pennsylvania 15260

E-mail address: neilan@pitt.edu 